\title{
Analysis of Reference Cigarette Smoke Yield Data from 21 Laboratories for 28 Selected Analytes as a Guide to Selection of New CORESTA Recommended Methods*
}

\author{
by \\ Steve Purkis ${ }^{1}$ and Michael Intorp ${ }^{2}$ \\ ${ }^{1}$ Imperial Tobacco Limited, Winterstoke Road, Bristol BS3 2LL, United Kingdom \\ ${ }^{2}$ Imperial Tobacco Group, Albert-Einstein-Ring 7, 22761 Hamburg, Germany
}

\section{SUMMARY}

Since 1999, the CORESTA Special Analytes Sub Group (SPA SG) has been working on the development of CORESTA Recommended Methods (CRMs) for the analysis of cigarette smoke components. All CRMs have been posted on the CORESTA website and several associated papers published. In this study, 21 laboratories shared data and in-house methodologies for 28 additional smoke components of regulatory interest to prioritise the development of further CRMs. Laboratories provided data, where available, from CORESTA monitor test pieces (CM6 and CM7) and Kentucky Reference Cigarettes (1R5F / 3R4F) covering the period 2010-2012 obtained under both the ISO 3308 and Health Canada Intense regimes.

Scant data were available on the CORESTA monitor test pieces and the Kentucky 1R5F reference. The greatest amount of data was obtained on the Kentucky 3R4F and this was used in the analyses described in this paper. SPA SG discussions provided invaluable insight into identifying causes and ways of reducing inter-laboratory variability which will be investigated in joint experiments before embarking on final collaborative studies using draft CRMs to obtain mean yields, repeatability and reproducibility values.

Phenolic compounds (phenol, 3 cresol isomers, hydroquinone, catechol and resorcinol) gave consistent results by liquid chromatography (LC) separation and fluorescence detection after extracting collected "tar" on a Cambridge filter pad (CFP). Yields were similar to those obtained by a derivatisation method followed by gas chromatography mass spectrometry (GC-MS) analysis. Similar ratios of phenols were also obtained from each method. Of the 28 studied analytes, the between-laboratory variability was lowest for the phenols.

Hydrogen cyanide was derivatised using various reagents and the colour development measured after continuous flow analysis (CFA) by ultra-violet absorbance. Although, methodologies gave reasonably consistent results, investigations on the trapping system and on differences in the application of the various colour complexes used for quantification with UV absorbance is required.

Ammonia analysis was carried out by ion chromatography (IC) followed by conductivity measurement and gave very similar results between laboratories. Yields were similar to those obtained by a derivatisation method followed by LC/MS-MS methodology. Optimal conditions for the separation of ammonium from interfering ions and minimizing artefactual ammonia formation from other smoke components need to be addressed during standardisation. Aromatic amine methods involved either LC/MS-MS separation and detection or derivatisation by one of two main reagents followed by GC-MS analysis. Yields were at similar but variable levels using these different techniques. It is currently unclear which method will be taken to a CRM. In general, four compounds were measured (1-amino naphthalene; 2-amino naphthalene; 3-amino biphenyl and 4-amino biphenyl) although two others were incorporated in methodologies used by 3 laboratories ( $o$-anisidine and $o$-toluidine).

Semi-volatiles (pyridine, quinoline and styrene) were often integrated with the selected volatiles method by measurement of the combination of CFP extracts and the contents of the impinger trapping system. 
Less data, obtained mainly by inductively-coupled plasma mass spectrometry (ICP-MS), were available on metals (cadmium, lead, arsenic, beryllium, cobalt, chromium, nickel, selenium and mercury) in smoke. Trace metals were the most variable of the studied smoke analytes. Optimisation of the digestion step to remove the organic matrix needs to be addressed.

As a consequence of this study and subsequent discussions within the Sub Group, it was decided to prioritise the development of CRMs for selected phenols followed by hydrogen cyanide and ammonia. [Beitr. Tabakforsch. Int. 26 (2014) 57-73]

\section{ZUSAMMENFASSUNG}

Seit 1999 arbeitet die CORESTA Special Analytes Sub Group (SPA SG) an der Entwicklung von CORESTA Recommended Methods (von der CORESTA empfohlene Methoden, CRM) zur Analyse von Bestandteilen im Zigarettenrauch. Alle CRM wurden auf der Internetseite der CORESTA veröffentlicht und mehrere Arbeiten wurden in diesem Zusammenhang publiziert. In der vorliegenden Studie haben 21 Labore ihre Daten und laborinternen Methoden für 28 zusätzliche Rauchbestandteile von Regulierungsinteresse eingebracht, um die Entwicklung weiterer CRM zu priorisieren. Soweit verfügbar, stellten die Labore Daten von CORESTA Monitor-Proben (CM6 und CM7) und KentuckyReferenzzigaretten (1R5F / 3R4F) aus dem Zeitraum 2010-2012 zur Verfügung, die nach ISO 3308 und Health Canada Intense-Protokollen gewonnen wurden.

$\mathrm{Zu}$ den CORESTA Monitor-Proben und zur KentuckyReferenzzigarette 1R5F standen nur spärliche Informationen zur Verfügung. Die meisten Daten lagen zur Kentucky 3R4F vor und diese wurden in den hier beschriebenen Analysen verwendet. Gespräche mit der SPA SG lieferten wertvolle Erkenntnisse für die Suche nach Ursachen und Möglichkeiten zur Reduzierung der Variabilität zwischen Laboren, die in gemeinsamen Experimenten weiter untersucht werden, bevor abschließende Kooperationsstudien mit CRM-Entwürfen zur Ermittlung von mittleren Ausbeuten, Wiederholgenauigkeit und Reproduzierbarkeit begonnen werden.

Phenolische Verbindungen (Phenol, 3 Kresol-Isomere, Hydrochinon, Katechol und Resorcin) zeigten nach Auftrennung durch Flüssigchromatographie (LC) und Fluoreszenzdetektion nach Extraktion von mit einem CambridgeFilter aufgefangenem “Teer” übereinstimmende Ergebnisse. Die Ausbeuten ähnelten denen, die mit einem Derivatisierungsverfahren gefolgt von GaschromatographieMassenspektrometrie (GC-MS)-Analyse gewonnen wurden. Jedes der Verfahren führte außerdem zu ähnlichen Anteilen von Phenolen. Von den 28 untersuchten Analyten war die Variabilität zwischen den Laboren für die Phenole am geringsten.

Wasserstoffcyanid wurde mit verschiedenen Reagenzien derivatisiert und nach Strömungsanalyse (CFA) wurde die Farbentwicklung durch UV-Absorption gemessen. Auch wenn die Verfahren relativ übereinstimmende Ergebnisse hervorbrachten, sind Untersuchungen zum Auffangsystem und zu Unterschieden in der Anwendung der verschiedenen Farbkomplexe für die Quantifizierung mit UV-Absorption notwendig.
Die Ammoniakanalyse wurde mittels Ionenchromatographie (IC), gefolgt von einer Leitfähigkeitsmessung, durchgeführt und ergab sehr ähnliche Ergebnisse zwischen den Laboren. Die Ausbeuten ähnelten denen, die mit einem Derivatisierungsverfahren gefolgt von Flüssigchromatographie/Tandem-Massenspektrometrie (GC-MS/MS) ermittelt wurden. Optimale Bedingungen für die Trennung von Ammonium von interierenden Ionen und die Minimierung einer artefakten Ammoniakbildung aus anderen Rauchbestandteilen müssen im Rahmen der Standardisierung untersucht werden.

Die Methoden für aromatische Amine umfassten entweder Auftrennung und Detektion durch LC/MS-MS oder Derivatisierung mit einem von zwei Hauptreagenzien gefolgt von einer GC-MS-Analyse. Bei Verwendung dieser verschiedenen Techniken lagen die Ausbeuten auf einem ähnlichen, aber variablen Niveau. Es ist derzeit unklar, welche Methode zu einer CRM entwickelt wird. Generell wurden vier Verbindungen gemessen (1-Aminonaphthalin, 2-Aminonaphthalin, 3-Aminobiphenyl und 4-Aminobiphenyl). Jedoch umfassten die Methoden von 3 Laboren zwei weitere Verbindungen ( $o$-Anisidin und $o$-Toluidin). Halbflüchtige Verbindungen (Pyridin, Chinolin und Styrol) wurden oft in die für flüchtige Verbindungen ausgewählte Methode der Messung der Kombination von CambridgeFilter-Extrakten und dem Inhalt des Impinger-Auffangsystems integriert.

Weniger Daten, hauptsächlich aus Massenspektrometrie mit induktiv gekoppeltem Plasma (ICP-MS), standen zu Metallen (Cadmium, Blei, Arsen, Beryllium, Kobalt, Chrom, Nickel, Selen und Quecksilber) im Rauch zur Verfügung. Spurenmetalle wiesen dabei von allen untersuchten Rauchanalyten die höchste Variabilität auf. Eine Optimierung des Verdauschritts zur Entfernung der organischen Matrix muss weiter untersucht werden.

Infolge dieser Studie und anschließender Erörterungen in der Sub Group wurde beschlossen, die Entwicklung von CRM für ausgewählte Phenole gefolgt von Wasserstoffcyanid und Ammoniak zu priorisieren. [Beitr. Tabakforsch. Int. 26 (2014) 57-73]

\section{RESUME}

Depuis 1999, le sous-groupe CORESTA " Special Analytes" (SG SPA) travaille sur le développement de méthodes recommandées par CORESTA (MRC) pour l'analyse des composants de la fumée de cigarette. Toutes les MRC ont été affichées sur le site Internet de CORESTA et plusieurs travaux associés ont été publiés. Dans la présente étude, 21 laboratoires ont partagé des données et des méthodologies internes pour 28 composants de fumée supplémentaires présentant un intérêt sur le plan réglementaire, dans le but de prioriser le développement de futures MRC. Des laboratoires ont fourni des données, là où ils en avaient, sur des échantillons de test sous surveillance de CORESTA (CM6 et CM7) et des cigarettes de référence Kentucky (1R5F / 3R4F), collectées pendant la période de 2010 à 2012 et obtenues avec les deux régimes de fumage intense d'ISO 3308 et de Health Canada.

Des données étaient disponibles en nombre réduit sur les échantillons de test sous surveillance de CORESTA et sur les 
cigarettes de référence Kentucky 1R5F. La plus grande quantité de données a été obtenue sur la cigarette Kentucky 3R4F et c'est ce qui a été utilisé dans les analyses décrites dans le présent rapport. Les discussions du SG SPA se sont soldées par de précieuses conclusions concernant l'identification des causes et des moyens de réduire la variabilité interlaboratoire, qui sera examinée au cours d'expérimentations communes avant de se lancer dans des études finales communes en utilisant les MRC ébauchées pour obtenir des rendements moyens ainsi que des valeurs de répétabilité et de reproductibilité.

Les composés phénoliques (phénol, 3 isomères du crésol, hydroquinone, catéchol et résorcinol) ont donné des résultats cohérents lors d'une séparation par chromatographie liquide (CL) et d'une détection par fluorescence après extraction du goudron sur un filtre Cambridge (FC). Les rendements ont été similaires à ceux obtenus par une méthode de dérivatisation suivie d'une analyse par chromatographie en phase gazeuse-spectroscopie de masse (CG-SM). Des ratios de phénol semblables ont également été obtenus par chaque méthode. Parmi les 28 analytes étudiés, la variabilité interlaboratoire était la plus basse pour le phénol.

Le cyanure d'hydrogène a été dérivatisé en utilisant des réactifs variés ainsi que le développement de la couleur mesuré après une analyse de flux continu (AFC) par absorbance UV. Bien que les méthodologies aient fourni des résultats d'une cohérence raisonnable, il est nécessaire d'étudier le système de piégeage et les différences dans l'application des divers complexes de couleur utilisés pour la quantification avec l'absorbance UV.

L'analyse de l'ammoniac a été effectuée par chromatographie ionique (CI) suivie d'une mesure de la conductivité et a donné des résultats très similaires entre les laboratoires. Les rendements ont été semblables à ceux obtenus au moyen d'une méthode de dérivatisation suivie d'une méthode de CL/SM-SM. Lors de la phase de normalisation, il faudra étudier les conditions optimales pour séparer l'ammonium des ions perturbateurs et minimiser la formation artéfactuelle d'ammoniac par d'autres composants de la fumée.

La méthode des acides aminés aromatiques a impliqué soit une séparation par CL/SM-SM et une détection ou une dérivatisation par l'un des deux réactifs principaux suivie d'une analyse par CG-SM. Les rendements ont présenté des niveaux similaires mais variables en fonction des différentes techniques utilisées. Il n'est pas encore clair quelle méthode sera prise pour élaborer une MRC. En général, quatre composés ont été mesurés (1-naphtalèneamine; 2-naphtalèneamine; 3-biphénylamine et 4-biphénylamine) bien que deux autres aient aussi été incorporés dans des méthodologies utilisées par 3 laboratoires ( $o$-anisidine et $o$-toluidine).

Des semi-volatiles (pyridine, quinoline et styrène) ont souvent été intégrés à la méthode des volatiles sélectionnés en mesurant la combinaison d'extraits du FC et les contenus du système de piégeage de l'impacteur.

Des données réduites, obtenues principalement par spectrométrie de masse à plasma à couplage inductif (SM$\mathrm{PCI}$ ), étaient disponibles pour les métaux (cadmium, plomb, arsenic, béryllium, cobalt, chrome, nickel, sélénium et mercure) présents dans la fumée. Les métaux traces présentaient la variabilité la plus élevée parmi les analytes de fumée étudiés. Il faudra étudier l'optimisation de l'étape de digestion pour éliminer la matrice organique.
Suite à cette étude et aux discussions ultérieures au sein du sous-groupe, il a été décidé de prioriser le développement de MRC pour des phénols sélectionnés, puis pour le cyanure d'hydrogène et l'ammoniac. [Beitr. Tabakforsch. Int. 26 (2014) 57-73]

\section{INTRODUCTION}

The CORESTA Special Analytes Sub Group (SPA SG) has now developed Recommended Methods (CRMs) for those compounds considered priority substances by the WHO (1). CRMs are available on benzo $[a]$ pyrene, CRM 58 (2); tobacco specific nitrosamines (TSNAs), CRMs 63 and 75 $(3,4)$; selected volatiles, CRM 70 (5) and selected carbonyls, CRM 74 (6). In the current versions, a range of cigarette types were smoked under both the ISO (7) and Health Canada Intense (HCI) (8) smoking regimes to provide mean yield, repeatability and reproducibility data in each case. In addition, several papers have been published providing more details on joint experiments concerning aromatic amines (9) and selected volatiles (10) and concerning collaborative studies on selected volatiles (11), selected carbonyls (12) and TSNAs (13) under ISO smoking. A short communication on benzo $[a]$ pyrene $(\mathrm{B}[a] \mathrm{P})$, selected volatiles and selected carbonyls has also been published on the work that updated the CRMs with data obtained under both ISO and HCI smoking regimes (14). Only the $\mathrm{B}[a] \mathrm{P}$ method has been developed into an ISO standard (15) and in 2013 the TSNA CRM 75 was raised as a new work item proposal for an ISO standard within ISO Technical Committee 126 on tobacco and tobacco products.

In 2006, SPA SG members shared yield data under the ISO smoking regime from Kentucky Reference Cigarettes for available smoke analytes as determined by their own inhouse methods (16). This showed that there was considerable variability in certain analyte yields and that those that had been standardised at that time were the most reproducible (B $[a]$ P and TSNAs). However, even these yields were still less reproducible than "tar", nicotine and CO. The present study describes an update for 28 smoke analytes that have not so far been taken to CORESTA CRMs but reflects current regulatory interest, for example, according to annual reporting in Canada (17) and within FDA regulations (18). Data and methodology were requested on the analytes listed in Table 1.

SPA SG members agreed to share both data and general

Table 1. Smoke analyte list.

\begin{tabular}{|c|c|c|}
\hline \multicolumn{2}{|c|}{$\begin{array}{l}\text { Substantial 3R4F data provided } \\
\text { for these analytes }\end{array}$} & $\begin{array}{c}\text { Minimal data provided } \\
\text { for these analytes }\end{array}$ \\
\hline Ammonia & Hydroquinone & Arsenic \\
\hline 1-Amino naphthalene & Catechol & Beryllium \\
\hline 2-Amino naphthalene & Resorcinol & Cobalt \\
\hline 3-Amino biphenyl & Pyridine & Chromium \\
\hline 4-Amino biphenyl & Quinoline & Nickel \\
\hline Hydrogen cyanide & Styrene & Selenium \\
\hline Phenol & Cadmium & Mercury \\
\hline o-Cresol & Lead & Manganese \\
\hline$p$-Cresol & & o-Anisidine \\
\hline$m$-Cresol & & o-Toluidine \\
\hline
\end{tabular}


Table 2. Summary of hydrogen cyanide methodology. Various colour complexes are developed for HCN determination and some published examples of such methodologies for HCN measurements in smoke are given herein $(19,20)$.

\begin{tabular}{|c|c|c|c|c|c|c|c|c|}
\hline \multirow[b]{2}{*}{ Lab } & \multirow[b]{2}{*}{ Machine } & \multicolumn{2}{|c|}{ No cigs per replicate } & \multicolumn{2}{|c|}{ Trapping system } & \multirow{2}{*}{$\begin{array}{l}\text { Derivatisation } \\
\text { with colour } \\
\text { reagent }\end{array}$} & \multicolumn{2}{|c|}{ Analytical equipment } \\
\hline & & ISO & $\mathrm{HCl}$ & $\begin{array}{c}\text { Filter / } \\
\text { diameter }(\mathrm{mm}) / \\
\text { pad treatment }^{\mathrm{a}}\end{array}$ & $\begin{array}{c}\text { Impingers / } \\
\text { trapping } \\
\text { solution } \\
\end{array}$ & & Equipment $^{\mathrm{b}}$ & $\begin{array}{c}\text { Detector / } \\
\text { wavelength }^{\mathrm{c}}\end{array}$ \\
\hline 1 & Linear & 3 & 1 & CFP / 44 & $1 / \mathrm{NaOH}$ & Yes & CFA & Spectrophotometer / $570 \mathrm{~nm}$ \\
\hline 2 & Linear & 5 & 3 & CFP / 44 & $1 / \mathrm{NaOH}$ & Yes & CFA & Spectrophotometer / $540 \mathrm{~nm}$ \\
\hline 3 & Linear & 5 & 2 & None & $2 / \mathrm{NaOH}$ & Yes & LC & MS-MS \\
\hline 4 & Linear & 4 & 4 & CFP / $44 / \mathrm{NaOH}$ & None & Yes & CFA & Spectrophotometer / $600 \mathrm{~nm}$ \\
\hline 5 & Linear & 4 & 2 & CFP / 44 & $1 / \mathrm{NaOH}$ & Yes & CFA & Spectrophotometer / $600 \mathrm{~nm}$ \\
\hline 6 & Linear & 4 & 3 & CFP / 44 & 1 & Yes & CFA & Spectrophotometer / $575 \mathrm{~nm}$ \\
\hline 7 & Linear & 3 & 2 & CFP / 44 & Silica gel & Yes & CFA & Spectrophotometer / $540 \mathrm{~nm}$ \\
\hline 8 & Linear & 4 & 4 & None & 1 & Yes & CFA & Spectrophotometer / $480 \mathrm{~nm}$ \\
\hline 9 & Rotary & 10 & 5 & None & 2 & Yes & LC & MS-MS \\
\hline 10 & Rotary & 5 & 5 & CFP / 92 & 2 & No & IC & Conductivity \\
\hline 11 & Linear & 5 & 3 & CFP / 44 & 2 & Yes & CFA & Spectrophotometer / $540 \mathrm{~nm}$ \\
\hline 12 & Rotary & 5 & 5 & CFP / 44 & $1 / \mathrm{NaOH}$ & Yes & CFA & Spectrophotometer / $540 \mathrm{~nm}$ \\
\hline 14 & Linear & 5 & 2 & CFP / 44 & 1 & Yes & CFA & Spectrophotometer / $630 \mathrm{~nm}$ \\
\hline 15 & Rotary & 5 & 2 & CFP / 92 & 1 & Yes & CFA & Spectrophotometer / $540 \mathrm{~nm}$ \\
\hline 16 & Linear & 3 & 2 & CFP / 44 & $1 / \mathrm{NaOH}$ & Yes & CFA & Spectrophotometer / $540 \mathrm{~nm}$ \\
\hline 17 & Linear & 3 & 1 & CFP / 44 & 1 & Yes & CFA & Spectrophotometer / $570 \mathrm{~nm}$ \\
\hline 18 & Rotary & 5 & 2 & CFP / 44 & 1 & Yes & CFA & Spectrophotometer / $600 \mathrm{~nm}$ \\
\hline 20 & Linear & 5 & 3 & CFP / 44 & $1 / \mathrm{NaOH}$ & Yes & CFA & Spectrophotometer / $570 \mathrm{~nm}$ \\
\hline 21 & Linear & 5 & 3 & CFP / 44 & 2 & Yes & LC & MS-MS \\
\hline
\end{tabular}

a $\quad$ CFP $=$ Cambridge filter pad

b $\quad$ CFA= continuous flow analysis; $I C=$ ion chromatography; LC = liquid chromatography

c $\mathrm{MS}=$ mass spectrometry

principles of their in-house methodologies for these analytes so that the next set of priority compounds could be identified for development of CRMs. In this way SPA SG might be able to choose either methodologies that might be easiest to standardise or those that might benefit the most from standardisation based on data and information provided by the 21 laboratories. It can be noted that only minimal numbers of data sets were obtained for 10 of the 28 smoke analytes (Table 1) particularly the trace metals.

\section{METHODOLOGY}

The participants used a range of numbers of cigarettes smoked per replicate, a mixture of rotary and linear machines for smoke collection and a range of methodologies for extraction, separation and detection. The main parameters and steps in the methodologies are highlighted below.

\section{Hydrogen cyanide}

In the case of hydrogen cyanide, the numbers of cigarettes smoked per replicate ranged from 3 to 10 for ISO and 1 to 5 for HCI smoking regimes. Five laboratories used rotary and 14 used linear smoking machines. Smoke was collected on CFPs in combination with either one or two impingers, derivatised with various reagents in preparation for continuous flow analysis by most laboratories. The different detection wavelengths shown are often an indication of the different derivatisation reagents used. The following methodological information given in Table 2 was collected from 19 participants.

\section{Selected aromatic amines}

The main components of the methodologies used by 18 laboratories to measure 1-amino naphthalene, 2-amino naphthalene, 3-amino biphenyl and 4-amino biphenyl are given in Table 3 . The numbers of cigarettes smoked per replicate ranged from 5 to 20 for ISO and 2 to 10 for HCI smoking regimes. Eight laboratories used rotary and 10 used linear smoking machines; eight laboratories used a method that involved derivatisation with pentafluoro propionic anhydride (PFPA) then GC-MS analysis and a further eight laboratories used a method that involved derivatisation with heptafluoro butyric anhydride (HFBA) then GC-MS analysis. LC-MS/MS separation and detection was used by two laboratories.

Previous joint experiments (9) had shown the use of similar methodologies although some laboratories had changed methodologies since the last study, for example, in the clean-up procedure. Laboratories 3, 6 and 12 incorporated $o$-anisidine and $o$-toluidine in the same methodology.

\section{Ammonia}

Ammonia methodology and analyte yields were received from 21 laboratories. The main components of the methodologies are given in Table 4. In common with other analytes, there was a wide range of numbers of cigarettes smoked per replicate ( 3 to 20 for ISO and 2 to 10 for HCI smoking regimes). Eight laboratories used rotary smoking machines and 13 used linear smoking machines. Separation was carried out by ion chromatography with conductivity detection in 18 laboratories. 
Table 3. Summary of aromatic amine methodology. Some methodological references have been given previously in the published literature (9).

\begin{tabular}{|c|c|c|c|c|c|c|}
\hline \multirow{2}{*}{ Lab } & \multirow{2}{*}{ Machine } & \multicolumn{2}{|c|}{ No cigs per replicate } & \multirow{2}{*}{$\begin{array}{l}\text { Derivatisation } \\
\text { agent }^{a}\end{array}$} & \multirow{2}{*}{$\begin{array}{l}\text { Derivatisation conditions } \\
\text { temp }\left({ }^{\circ} \mathrm{C}\right) / \text { time }(\min )\end{array}$} & \multirow{2}{*}{$\begin{array}{c}\text { Analytical separation / } \\
\text { detection }^{\mathrm{b}}\end{array}$} \\
\hline & & ISO & $\mathrm{HCl}$ & & & \\
\hline 1 & Linear & 5 & 3 & PFPA / TMA & Ambient / 60 & GC-MS \\
\hline 2 & Linear & 5 & 3 & PFPA / TMA & $80 / 30$ & GC-MS \\
\hline 3 & Linear & 5 & 2 & HFBA & $80 / 30$ & GC-MS \\
\hline 4 & Linear & 20 & - & PFPA / TMA & Ambient / 40 & GC-MS \\
\hline 5 & Linear & 20 & 10 & None & - & LC / MS-MS \\
\hline 6 & Rotary & 20 & 10 & HFBA & $80 / 30$ & GC-MS \\
\hline 7 & Linear & 5 & 3 & None & - & LC / MS-MS \\
\hline 8 & Rotary & 10 & 3 & HFBA & Ambient / 40 & GC-MS \\
\hline 9 & Rotary & 10 & 5 & HFBA & $80 / 30$ & GC-MS \\
\hline 11 & Rotary & 20 & 10 & HFBA & $80 / 30$ & GC-MS \\
\hline 12 & Rotary & 10 & 5 & PFPA / TMA & Ambient / >120 & GC-MS \\
\hline 14 & Rotary & 10 & 4 & HFBA & $80 / 30$ & GC-MS \\
\hline 15 & Linear & 5 & 2 & PFPA / TMA & Ambient / 30 & GC-MS \\
\hline 16 & Rotary & 10 & 5 & HFBA & Ambient / 30 & GC-MS \\
\hline 18 & Linear & 5 & 2 & PFPA / TMA & Ambient / 45 & GC-MS \\
\hline 19 & Rotary & 10 & 5 & PFPA & Ambient / >120 & GC-MS \\
\hline 20 & Linear & 5 & 3 & HFBA & $80 / 30$ & GC-MS \\
\hline 21 & Linear & 5 & - & PFPA & Ambient / 120 & GC-MS \\
\hline
\end{tabular}

a $\mathrm{HFBA}=$ heptafluoro butyric anhydride; PFPA = pentafluoro propionic anhydride; TMA = trimethylamine

b $\quad \mathrm{GC}=$ gas chromatography; $\mathrm{MS}=$ mass spectrometry

Table 4. Summary of ammonia methodology. General principles for the IC (21) and LC (22) methods have been described.

\begin{tabular}{|c|c|c|c|c|c|c|c|c|c|c|}
\hline \multirow[b]{2}{*}{ Lab } & \multirow[b]{2}{*}{ Machine } & \multicolumn{2}{|c|}{ No cigs per replicate } & \multirow{2}{*}{\begin{tabular}{|c|} 
Trapping system \\
$\begin{array}{c}\text { Impingers / acid in } \\
\text { trapping solution }\end{array}$ \\
\end{tabular}} & \multirow[b]{2}{*}{$\begin{array}{c}\text { Filtration } \\
\text { step }\end{array}$} & \multirow[b]{2}{*}{$\begin{array}{c}\text { Derivatisation } \\
\text { agent }\end{array}$} & \multicolumn{2}{|c|}{ Analytical conditions } & \multirow[b]{2}{*}{ Detection $^{\mathrm{b}}$} & \multirow[b]{2}{*}{$\begin{array}{c}\text { Calibration } \\
\text { curve }\end{array}$} \\
\hline & & ISO & $\mathrm{HCl}$ & & & & $\begin{array}{c}\text { Autosampler / } \\
\text { temp }\left({ }^{\circ} \mathrm{C}\right)\end{array}$ & Equipment $^{a}$ & & \\
\hline 1 & Linear & 5 & 3 & $\begin{array}{c}2 \text { / methane } \\
\text { sulphonic acid }\end{array}$ & Yes & None & Yes / 10 & IC / isocratic & Conductivity & Linear \\
\hline 2 & Linear & 3 & 2 & $1 /$ malic acid & Yes & None & Yes $/<10$ & IC / isocratic & Conductivity & Quadratic \\
\hline 3 & Linear & 5 & 2 & $1 / \mathrm{HCl}$ & Yes & Dansyl chloride & No & LC / isocratic & MS-MS & Linear \\
\hline 4 & Linear & 3 & 3 & $1 /$ unspecified & Yes & None & No & IC / isocratic & Conductivity & Quadratic \\
\hline 5 & Linear & 4 & 2 & $1 /$ unspecified & Yes & None & No & IC / isocratic & Conductivity & Linear \\
\hline 6 & Linear & 5 & 3 & $1 /$ unspecified & Yes & None & Yes / ambient & IC / isocratic & Conductivity & Quadratic \\
\hline 7 & Rotary & 10 & 10 & 2 / unspecified & Yes & None & No & IC / isocratic & Conductivity & Quadratic \\
\hline 8 & Linear & 5 & 4 & $1 /$ unspecified & Yes & None & No & IC / isocratic & Conductivity & Linear \\
\hline 9 & Rotary & 10 & 5 & 2 / unspecified & Yes & Dansyl chloride & Yes / 17 & LC / isocratic & MS-MS & Linear \\
\hline 10 & Rotary & 20 & 10 & $1 /$ unspecified & Yes & None & No & IC / isocratic & Conductivity & Linear \\
\hline 11 & Linear & 3 & 3 & $1 /$ unspecified & Yes & None & Yes / 10 & IC / isocratic & Conductivity & Quadratic \\
\hline 12 & Rotary & 5 & 3 & $2 / \mathrm{H}_{2} \mathrm{SO}_{4}$ & Yes & None & Yes / 4 & IC / gradient & Conductivity & Quadratic \\
\hline 13 & Linear & 3 & 3 & $1 / \mathrm{HCl}$ & Yes & None & Yes / ambient & IC / isocratic & Conductivity & Quadratic \\
\hline 14 & Rotary & 10 & 3 & 2 / unspecified & Yes & None & Yes / 4 & IC / isocratic & Conductivity & Quadratic \\
\hline 15 & Rotary & 10 & 5 & 2 / unspecified & Yes & None & Yes / 5 & IC / isocratic & Conductivity & Quadratic \\
\hline 16 & Rotary & 10 & 5 & 2 / unspecified & Yes & None & Yes / 5 & IC / isocratic & Conductivity & Quadratic \\
\hline 17 & Linear & 5 & 3 & 2 / unspecified & Yes & None & Yes / 10 & IC / isocratic & Conductivity & Linear \\
\hline 18 & Rotary & 10 & 2 & $1 /$ unspecified & Yes & None & No & IC / isocratic & Conductivity & Quadratic \\
\hline 19 & Linear & 3 & 3 & $1 /$ unspecified & Yes & None & Yes / ambient & IC / isocratic & Conductivity & Quadratic \\
\hline 20 & Linear & 5 & 3 & $1 /$ unspecified & Yes & None & Yes / 5 & IC / gradient & Conductivity & Linear \\
\hline 21 & Linear & 5 & 3 & 1 / unspecified & Yes & Dansyl chloride & Yes / 4 & LC / isocratic & MS-MS & Linear \\
\hline
\end{tabular}

a IC = ion chromatography; LC = liquid chromatography

b $\quad$ MS-MS = mass spectrometry - mass spectrometry 
Table 5. Summary of phenolic methodology. General principles for the HPLC (23) and GC (24) methods have been described.

\begin{tabular}{|c|c|c|c|c|c|c|c|c|}
\hline \multirow{2}{*}{ Lab } & \multirow{2}{*}{ Machine } & \multicolumn{2}{|c|}{ No cigs per replicate } & \multirow{2}{*}{ CFP extraction } & \multirow{2}{*}{ Filtration } & \multirow{2}{*}{ Derivatisation $^{a}$} & \multirow{2}{*}{ Separation ${ }^{\mathrm{b}}$} & \multirow{2}{*}{ Detection } \\
\hline & & ISO & $\mathrm{HCl}$ & & & & & \\
\hline 1 & Linear & 5 & 3 & Water / acetic acid / methanol & Yes & No & HPLC & Fluorescence \\
\hline 2 & Linear & 5 & 3 & Water / acetic acid & Yes & No & HPLC & Fluorescence \\
\hline 3 & Linear & 5 & 2 & 2-Butanone & No & BSTFA & $\mathrm{GC}$ & MS \\
\hline 4 & Linear & 4 & - & Water / acetic acid & Yes & No & HPLC & Fluorescence \\
\hline 5 & Linear & 4 & 2 & Water / acetic acid & Yes & No & HPLC & Fluorescence \\
\hline 6 & Linear & 5 & 3 & Water / acetic acid & Yes & No & HPLC & Fluorescence \\
\hline 7 & Linear & 5 & 3 & Propanol & Yes & No & HPLC & Fluorescence \\
\hline 8 & Linear & 4 & 3 & Dimethyl formamide & No & BSTFA & GC & MS \\
\hline 9 & Rotary & 10 & 10 & 2-Butanone & No & BSTFA & GC & MS \\
\hline 11 & Linear & 5 & 3 & $t$-Butyl methyl ether & No & BSTFA & $\mathrm{GC}$ & MS \\
\hline 12 & Linear & 5 & 3 & Water / acetic acid & Yes & No & HPLC & Fluorescence \\
\hline 13 & Rotary & 5 & 3 & Water / hydrochloric acid & Yes & No & HPLC & Fluorescence \\
\hline 14 & Linear & 5 & 2 & Water / acetic acid & Yes & No & HPLC & Fluorescence \\
\hline 15 & Rotary & 10 & 5 & Water / acetic acid & Yes & No & HPLC & Fluorescence \\
\hline 16 & Rotary & 4 & 2 & Water / acetic acid & Yes & No & HPLC & Fluorescence \\
\hline 17 & Linear & 5 & 3 & Water / acetic acid / methanol & Yes & No & HPLC & Fluorescence \\
\hline 18 & Linear & 5 & 2 & Water / acetic acid & Yes & No & HPLC & Fluorescence \\
\hline 19 & Rotary & 20 & 10 & Water / acetic acid & Yes & No & HPLC & Fluorescence \\
\hline 20 & Linear & 5 & 2 & Water / acetic acid / methanol & Yes & No & HPLC & Fluorescence \\
\hline 21 & Linear & 5 & - & Water / acetic acid / methanol & No & No & HPLC & Fluorescence \\
\hline
\end{tabular}

a $\quad$ BSTFA $=$ N,O-Bis(trimethylsilyl)trifluoroacetamide

b $\mathrm{HPLC}=$ high performance liquid chromatography

Three laboratories carried out derivatisation then used LCMS/MS for separation and detection. For each of these laboratories, a CFP with holder was put in line before the impinger(s).

\section{Selected phenolic compounds}

Twenty laboratories carried out measurements on seven phenolic compounds (phenol; $o$-cresol; $p$-cresol; $m$-cresol; hydroquinone; resorcinol and catechol) according to methodologies summarised in Table 5. The numbers of cigarettes smoked per replicate ranged from 4 to 10 for the ISO and 2 to 10 for the HCI smoking regime. Five laboratories used a rotary smoking machine and the others used linear machines. After smoke collection four laboratories derivatised the smoke using $\mathrm{N}, \mathrm{O}$-Bis(trimethylsilyl)trifluoroacetamide (BSTFA) then separated and measured components by GC-MS. The other laboratories carried out HPLC separation of the smoke extract with fluorescence detection.

\section{Selected semi-volatile compounds}

The studied analytes were pyridine; quinoline and styrene. The methodologies are summarised in Table 6 and were sometimes incorporated in the collection set up for the selected volatiles using methods based on CRM 70 (5). Nine laboratories used a rotary machine for smoke collection and 10 used a linear machine. Most participants collected on a CFP in combination with a range of one to three cooled impingers although three laboratories used a trapping column. One collected semi-volatiles on a CFP in combination with a Tedlar bag. Each laboratory then carried out separation and detection by GC-MS.

\section{Selected metals}

Several trace metals are of regulatory interest including cadmium, lead, arsenic, chromium nickel, selenium, beryllium, cobalt, manganese and mercury. Data was obtained from 10 laboratories. Table 7 indicates that most laboratories used electrostatic precipitation for smoke collection. Nine of the 10 laboratories used rotary smoking machines. Various digestion methods were used to prepare the collected smoke samples for analysis but ion-coupled plasma - mass spectrometry (ICP-MS) detection was applied by all laboratories except one which used atomic absorption.

Where given, mercury methodology was described differently. For example, Laboratories 1, 8 and 14 used potassium permanganate as the extraction solution. Laboratory 1 used a flow injection mercury system for analysis and detection. Laboratory 8 used a charge couple device for detection and Laboratory 14 used a cold vapour atomic absorption spectrometry method.

\section{DATA ANALYSIS}

Laboratories were requested to provide data from reference cigarettes, where available. This included CORESTA monitor test pieces (CM6 and CM7) and Kentucky Reference Cigarettes (1R5F / 3R4F) covering the period 2010-2012. Their data was coded anonymously. The last ten data points analysed by the laboratory and the time period over which they were carried out were requested.

Table 8 summarises the data submitted. It can be observed that only limited data was available and provided on the CM6, CM7 and KY1R5F products. 
Table 6. Summary of semi-volatiles methodology. The general principles of the methodology have been described within the selected volatiles method $(5,11)$.

\begin{tabular}{|c|c|c|c|c|c|c|c|}
\hline \multirow{2}{*}{ Lab } & \multirow{2}{*}{ Machine } & \multicolumn{2}{|c|}{ No cigs per replicate } & \multirow{2}{*}{ CFP trapping } & \multirow{2}{*}{ Impingers / trapping solvent ${ }^{a}$} & \multirow{2}{*}{ Filtration } & \multirow{2}{*}{$\begin{array}{l}\text { Separation and } \\
\text { detection }^{\mathrm{b}}\end{array}$} \\
\hline & & ISO & $\mathrm{HCl}$ & & & & \\
\hline 1 & Linear & 5 & 3 & Yes & $1 /$ methanol & Yes & GC-MS \\
\hline 2 & Linear & 5 & 3 & Yes & $1 /$ methanol & Yes & GC-MS \\
\hline 3 & Linear & 5 & 2 & Yes & 2 / methanol & Yes & GC-MS \\
\hline 4 & Linear & 4 & - & Yes & "XAD" absorbent tube & Yes & GC-MS \\
\hline 5 & Linear & 4 & 2 & Yes & "XAD" absorbent tube & No & GC-MS \\
\hline 6 & Linear & 5 & 3 & Yes & $1 /$ methanol & No & GC-MS \\
\hline 7 & Linear & 5 & 3 & Yes & "XAD" absorbent tube & No & GC-MS \\
\hline 8 & Rotary & 10 & 3 & Yes & 2 / isopropanol / hexane & No & GC-MS \\
\hline 9 & Rotary & 10 & 10 & Yes & 2 / methanol & Yes & GC-MS \\
\hline 11 & Linear & 5 & 3 & Yes & 2 / methanol & No & GC-MS \\
\hline 12 & Rotary & 20 & 10 & Yes & $2 /$ methanol & No & GC-MS \\
\hline 13 & Rotary & 20 & 10 & Yes & 2 / methanol / hexane / ethyl acetate & Yes & GC-MS \\
\hline 14 & Rotary & 20 & 10 & Yes & $2 /$ methanol & No & GC-MS \\
\hline 15 & Rotary & 20 & 10 & Yes & 2 / methanol & Yes & GC-MS \\
\hline 16 & Rotary & 10 & 4 & Yes & $3 /$ methanol & Yes & GC-MS \\
\hline 17 & Linear & 5 & 3 & Yes & $1 /$ methanol / triethylamine & Yes & GC-MS \\
\hline 18 & Rotary & 10 & 5 & Yes & $1 /$ methanol & Yes & GC-MS \\
\hline 20 & Rotary & 20 & 10 & Yes & Tedlar bag & No & GC-MS \\
\hline 21 & Linear & 5 & 3 & Yes & $1 /$ methanol & Yes & GC-MS \\
\hline
\end{tabular}

a "XAD" = a type of absorbent resin

b GC-MS = gas chromatography - mass spectrometry

Table 7. Summary of metals methodology. General principles for these methods have been described (25).

\begin{tabular}{|c|c|c|c|c|c|c|c|}
\hline \multirow{2}{*}{ Lab } & \multirow{2}{*}{ Machine } & \multicolumn{2}{|c|}{ No cigs per replicate } & \multirow{2}{*}{ Trap $^{a}$} & \multirow{2}{*}{$\begin{array}{l}\text { Trapping and digestion } \\
\text { solutions }\end{array}$} & \multirow{2}{*}{ Digestion } & \multirow{2}{*}{ Detection } \\
\hline & & ISO & $\mathrm{HCl}$ & & & & \\
\hline 1 & Rotary & 20 & 10 & EP & $\mathrm{HNO}_{3} / \mathrm{H}_{2} \mathrm{O}_{2}$ & Digestion block & ICP-MS \\
\hline 2 & Rotary & 20 & 10 & EP & Methanol / $\mathrm{HNO}_{3} / \mathrm{H}_{2} \mathrm{O}_{2}$ & Digestion block & ICP-MS \\
\hline 3 & Rotary & 20 & 10 & EP & $\mathrm{HNO}_{3} / \mathrm{H}_{2} \mathrm{O}_{2}$ & Microwave & ICP-MS \\
\hline 7 & Linear & 10 & 5 & 2 impingers & $\mathrm{HNO}_{3}$ & Microwave & ICP-MS \\
\hline 8 & Rotary & 20 & 10 & EP & Methanol / $\mathrm{HNO}_{3} / \mathrm{H}_{2} \mathrm{O}_{2}$ & Microwave & ICP-MS \\
\hline 10 & Rotary & 20 & 10 & $\mathrm{EP}+1$ impinger & $\mathrm{HNO}_{3}$ & Microwave & AA \\
\hline 12 & Rotary & 20 & 10 & $\mathrm{EP}+1$ impinger & Methanol / $\mathrm{HNO}_{3} / \mathrm{H}_{2} \mathrm{O}_{2}$ & Microwave & ICP-MS \\
\hline 14 & Rotary & 20 & 10 & EP & Methanol / $\mathrm{HNO}_{3}$ & No digestion & ICP-MS \\
\hline 16 & Rotary & 20 & 10 & EP & $\mathrm{HNO}_{3}$ & Microwave & ICP-MS \\
\hline 20 & Rotary & 20 & 10 & $\mathrm{EP}$ & Methanol / $\mathrm{HNO}_{3}$ & Microwave & ICP-MS \\
\hline
\end{tabular}

a $\quad E P=$ electrostatic precipitation

b $\mathrm{AA}=$ atomic absorption; ICP-MS = ion-coupled plasma - mass spectrometry

The most widely studied reference product (3R4F) was selected for data comparisons in this paper. All received data for each analyte were used in this analysis and no outlier analysis was performed or data excluded. Mean yields from each laboratory using their preferred methodologies for 3R4F cigarettes are given in Table 9 under ISO smoking and in Table 10 under HCI smoking conditions (for both tables see pages 64 and 65). The individual mean yields and the overall mean yield between laboratories and the associated coefficient of variation expressed as a percentage are also provided. Table 9 also gives the data variability (shown as the coefficient of variation) obtained in the previous study (16) on $2 \mathrm{R} 4 \mathrm{~F}$ cigarettes under ISO smoking for comparative purposes with the 3R4F data. Data was collected over time periods ranging from under one month to 13 months so within-laboratory variability will reflect lesser or greater temporal effects. The time periods in months over which the different analytes were measured are given in Table 11. Figures 1 to 11 show the range of values as minimum and maximum yields in the "error" bars from the 10 replicates reflecting temporal variability for each laboratory and for selected analytes in each group.

\section{RESULTS AND DISCUSSION}

Some representative examples of mean, maximum and minimum yields for 3R4F cigarettes from each analysis group are given in Figures 1 to 11. Overall, the variability of data between different laboratories running their own methodologies has reduced since the previous study (16) as shown by the coefficient of variance values given in the two right hand columns in Table 9. This might be due to the laboratories becoming more proficient and also sharing data and experience through a collaborative forum such as the 
Table 8. Summary of datasets received for most $(\bullet \bullet \bullet)$, some $(\circ \circ)$ or no $(-)$ analytes.

\begin{tabular}{|c|c|c|c|c|c|c|c|c|}
\hline \multirow{2}{*}{ Lab } & \multicolumn{2}{|c|}{$3 R 4 F$} & \multicolumn{2}{|c|}{ 1R5F } & \multicolumn{2}{|c|}{ CM7 } & \multicolumn{2}{|c|}{ CM6 } \\
\hline & ISO & $\mathrm{HCl}$ & ISO & $\mathrm{HCl}$ & ISO & $\mathrm{HCl}$ & ISO & $\mathrm{HCl}$ \\
\hline 1 & $\bullet \bullet \bullet$ & $\bullet \bullet \bullet$ & - & - & - & - & - & - \\
\hline 2 & $\bullet \bullet \bullet$ & $\bullet \bullet$ & - & - & - & - & - & - \\
\hline 3 & $\bullet \bullet \bullet$ & $\bullet \bullet \bullet$ & $\bullet \bullet \bullet$ & - & - & - & - & - \\
\hline 4 & $\circ \circ$ & $\circ \circ$ & $\circ \circ$ & - & - & - & $\circ \circ$ & - \\
\hline 5 & ०० & $0 \circ$ & - & - & - & - & ० ० & - \\
\hline 6 & $\bullet \bullet \bullet$ & $\bullet \bullet \bullet$ & - & - & - & - & - & - \\
\hline 7 & $\bullet \bullet \bullet$ & $\bullet \bullet$ & - & - & - & - & - & - \\
\hline 8 & $\bullet \bullet \bullet$ & $\bullet \bullet$ & $\bullet \bullet$ & $\bullet \bullet \bullet$ & $\bullet \bullet \bullet$ & $\bullet \bullet \bullet$ & - & - \\
\hline 9 & $\bullet \bullet \bullet$ & $\bullet \bullet$ & - & - & - & - & - & - \\
\hline 10 & $\circ \circ$ & $\circ \circ$ & - & - & - & - & - & - \\
\hline 11 & $\bullet \bullet \bullet$ & $\bullet \bullet$ & - & - & - & - & - & - \\
\hline 12 & $\bullet \bullet \bullet$ & - & - & - & - & - & - & - \\
\hline 13 & - & - & $\circ \circ$ & $\circ \circ$ & - & - & - & - \\
\hline 14 & $\bullet \bullet \bullet$ & $\bullet \bullet$ & - & - & - & - & - & - \\
\hline 15 & $\bullet \bullet \bullet$ & $\bullet \bullet$ & - & - & - & - & - & - \\
\hline 16 & $\bullet \bullet \bullet$ & $\bullet \bullet \bullet$ & $\bullet \bullet \bullet$ & $\bullet \bullet \bullet$ & - & - & - & - \\
\hline 17 & $\bullet \bullet \bullet$ & $\circ \circ$ & - & - & - & - & - & - \\
\hline 18 & $\bullet \bullet \bullet$ & $\bullet \bullet$ & $\bullet \bullet$ & $\bullet \bullet \bullet$ & $\bullet \bullet \bullet$ & $\bullet \bullet \bullet$ & $\bullet \bullet$ & $\bullet \bullet \bullet$ \\
\hline 19 & - & - & - & - & - & - & $\circ \circ$ & - \\
\hline 20 & $\bullet \bullet \bullet$ & $\bullet \bullet \bullet$ & - & - & - & - & - & - \\
\hline 21 & ०० & $\circ \circ$ & ०० & ०० & - & - & - & - \\
\hline
\end{tabular}

Table 11. Time period (months) for $3 \mathrm{R} 4 \mathrm{~F}$ reference data collection.

\begin{tabular}{|c|c|c|c|c|c|c|c|c|c|c|c|c|}
\hline \multirow{3}{*}{ Lab } & \multicolumn{12}{|c|}{ Time (months) } \\
\hline & \multicolumn{2}{|c|}{ Ammonia } & \multicolumn{2}{|c|}{ Aromatic amines } & \multicolumn{2}{|c|}{$\mathrm{HCN}$} & \multicolumn{2}{|c|}{ Phenols } & \multicolumn{2}{|c|}{ Semi-volatiles } & \multicolumn{2}{|c|}{ Trace metals } \\
\hline & $\mathrm{ISO}^{\mathrm{a}}$ & $\mathrm{HCl}$ & ISO & $\mathrm{HCl}$ & ISO & $\mathrm{HCl}$ & ISO & $\mathrm{HCl}$ & ISO & $\mathrm{HCl}$ & ISO & $\mathrm{HCl}$ \\
\hline 1 & 3 & 5 & 6 & 6 & 1 & 1 & 3 & 12 & 4 & 12 & 5 & 1 \\
\hline 2 & 13 & 6 & 12 & 12 & 6 & 6 & 12 & 12 & 12 & 12 & 12 & - \\
\hline 3 & 9 & 3 & 11 & 1 & 2 & 3 & 11 & 1 & 12 & 1 & 12 & 2 \\
\hline 4 & $\mathrm{n} / \mathrm{a}$ & $\mathrm{n} / \mathrm{a}$ & - & - & $\mathrm{n} / \mathrm{a}$ & $\mathrm{n} / \mathrm{a}$ & $\mathrm{n} / \mathrm{a}$ & - & $\mathrm{n} / \mathrm{a}$ & - & - & - \\
\hline 5 & $\mathrm{n} / \mathrm{a}$ & $\mathrm{n} / \mathrm{a}$ & $\mathrm{n} / \mathrm{a}$ & $\mathrm{n} / \mathrm{a}$ & $\mathrm{n} / \mathrm{a}$ & $\mathrm{n} / \mathrm{a}$ & $\mathrm{n} / \mathrm{a}$ & - & $\mathrm{n} / \mathrm{a}$ & - & - & - \\
\hline 6 & 9 & 1 & 1 & 1 & 1 & - & 1 & 1 & 5 & 4 & 6 & - \\
\hline 7 & 3 & 3 & 2 & 2 & 2 & 2 & - & - & 2 & 2 & 2 & 6 \\
\hline 8 & 1 & 1 & 6 & 1 & 1 & 1 & 1 & 6 & 2 & 6 & - & 2 \\
\hline 9 & 8 & 3 & 11 & 1 & 8 & 4 & 2 & 3 & 10 & 2 & 2 & 2 \\
\hline 10 & 2 & 2 & - & - & 2 & 2 & - & - & 2 & - & - & - \\
\hline 11 & 3 & 3 & 1 & 2 & 1 & 1 & 1 & 2 & 1 & 1 & 4 & - \\
\hline 12 & 3 & 3 & 4 & 4 & 3 & 3 & 4 & 4 & 4 & 4 & 2 & 4 \\
\hline 14 & 2 & 4 & 2 & 2 & 2 & 4 & 2 & 2 & 2 & 4 & 2 & 3 \\
\hline 15 & 3 & 2 & 1 & 1 & 3 & 2 & 4 & 1 & 2 & 2 & & - \\
\hline 16 & 1 & 1 & 1 & 1 & 1 & 1 & 1 & 1 & 1 & 1 & 1 & 1 \\
\hline 17 & 1 & - & - & - & 3 & 1 & 1 & - & 1 & - & 3 & - \\
\hline 18 & 1 & 1 & 1 & 1 & 1 & 1 & 1 & 1 & 7 & 1 & - & 5 \\
\hline 20 & 2 & 5 & 1 & 2 & 1 & 1 & 3 & 8 & 1 & 7 & 7 & 2 \\
\hline 21 & - & - & $\mathrm{n} / \mathrm{a}$ & - & - & - & $\mathrm{n} / \mathrm{a}$ & - & $\mathrm{n} / \mathrm{a}$ & $\mathrm{n} / \mathrm{a}$ & - & - \\
\hline
\end{tabular}

a $\quad \mathrm{n} / \mathrm{a}=$ timeframe not available; $-\mathbf{-}=$ no data provided by lab

CORESTA SPA SG. It has recently been estimated that 3R4F temporal within-laboratory yield variability under ISO smoking is approximately $10 \%$ for the analytes that have already been taken to recommended methods (26). Results in this study on other analytes across a range of laboratories generally agree with this observation with a few exceptions. Greater levels of between laboratory variability are likely to be caused by differences in the different methodological steps such as smoke collection, extraction and work-up, separation and detection. 


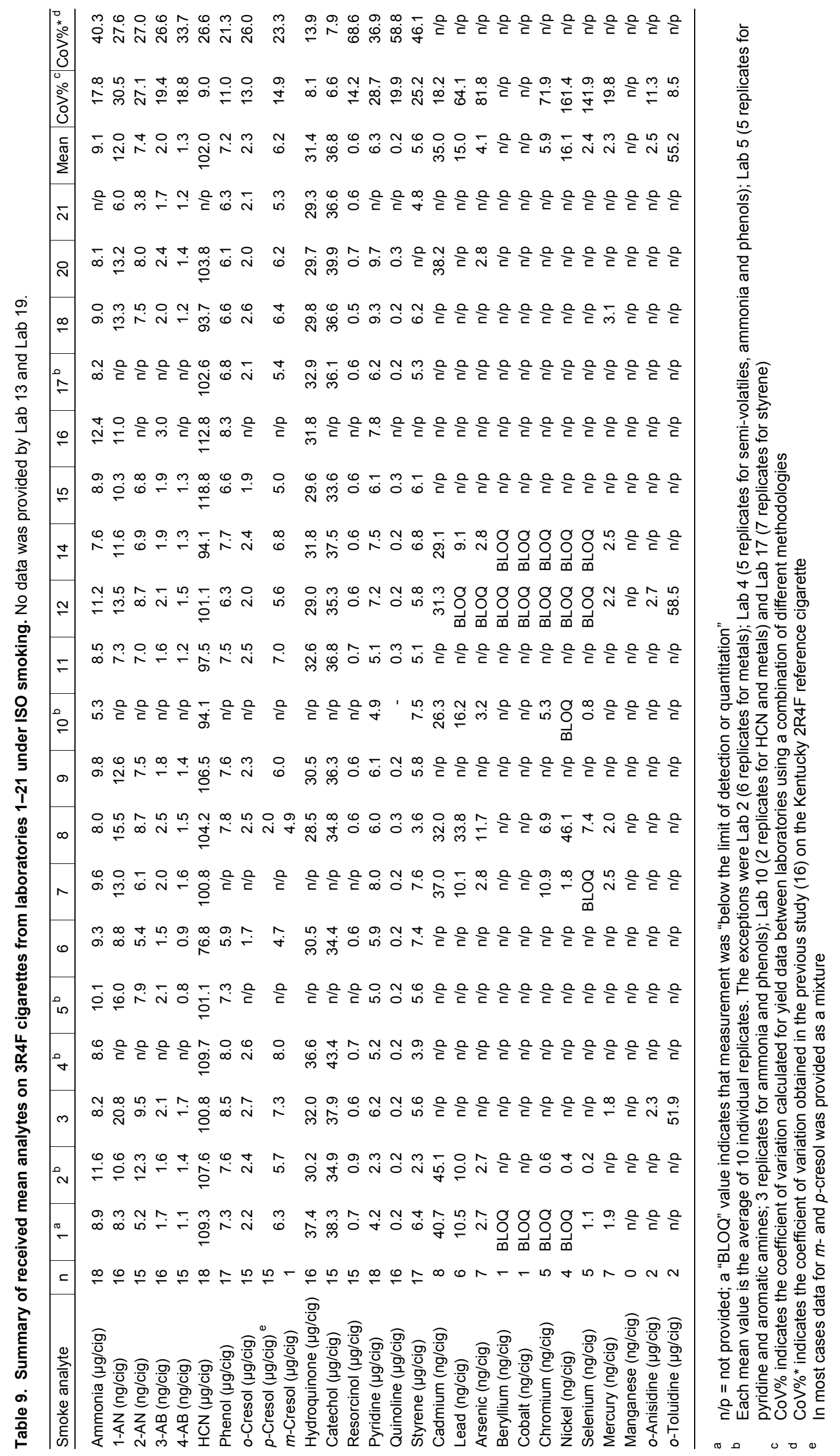




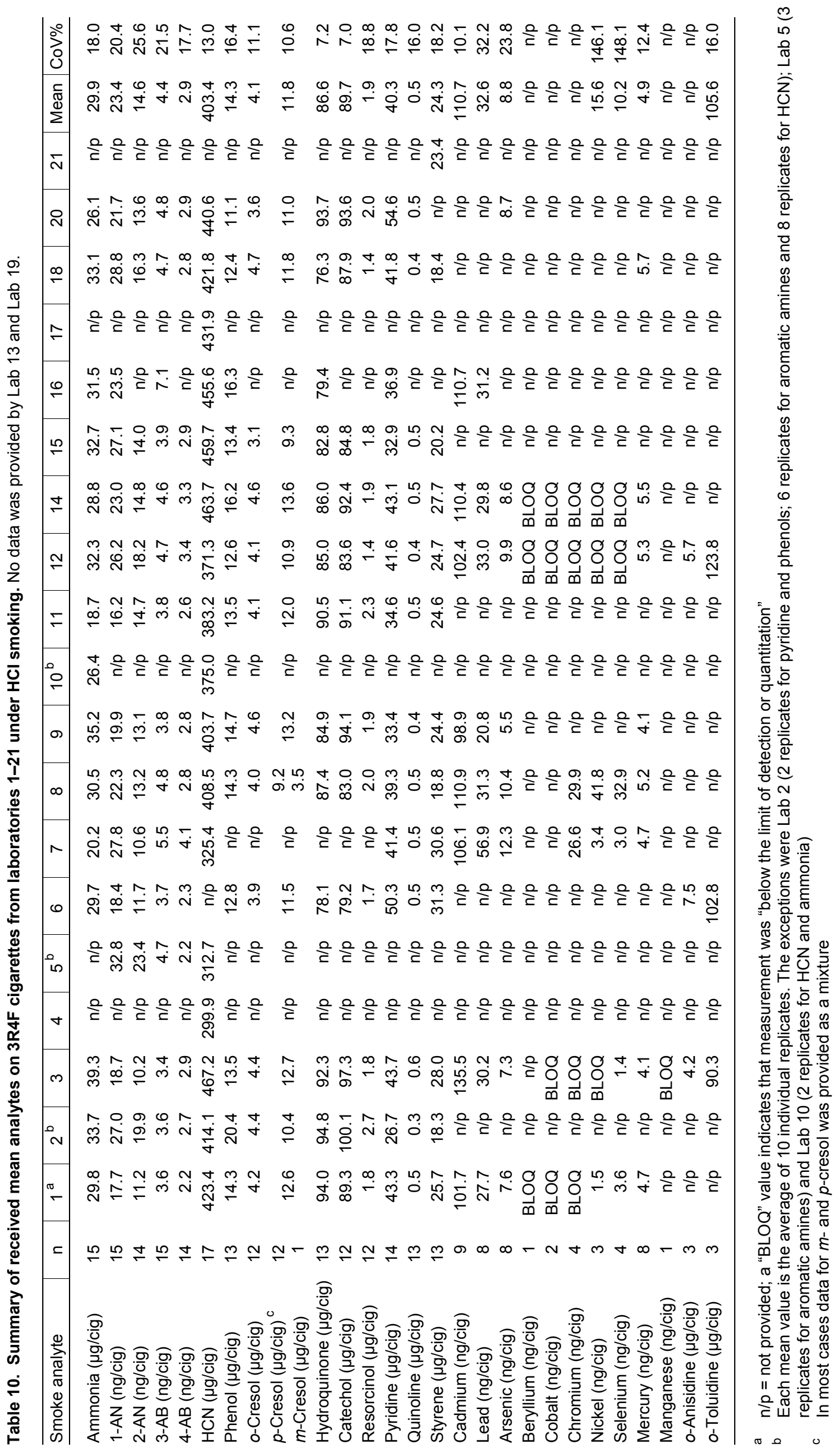




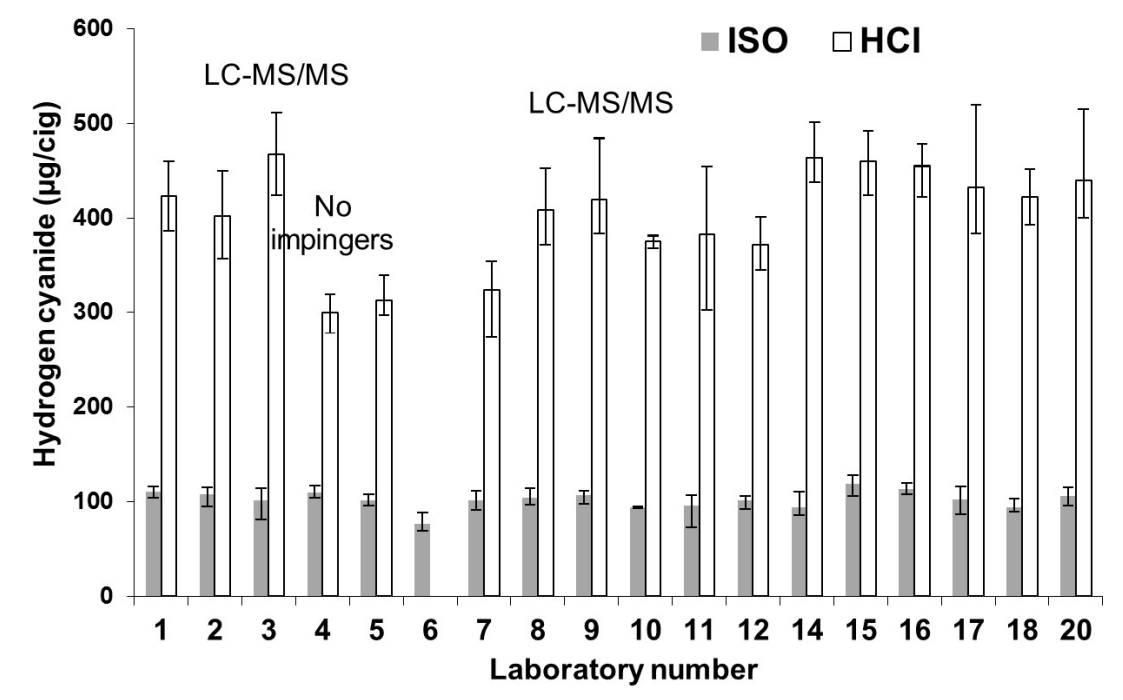

Figure 1. Hydrogen cyanide yields in Kentucky 3R4F mainstream smoke. LC-MS/MS = liquid chromatography - mass spectrometry / mass spectrometry. Maximum and minimum yield values from the 10 replicates are given in the "error" bars.

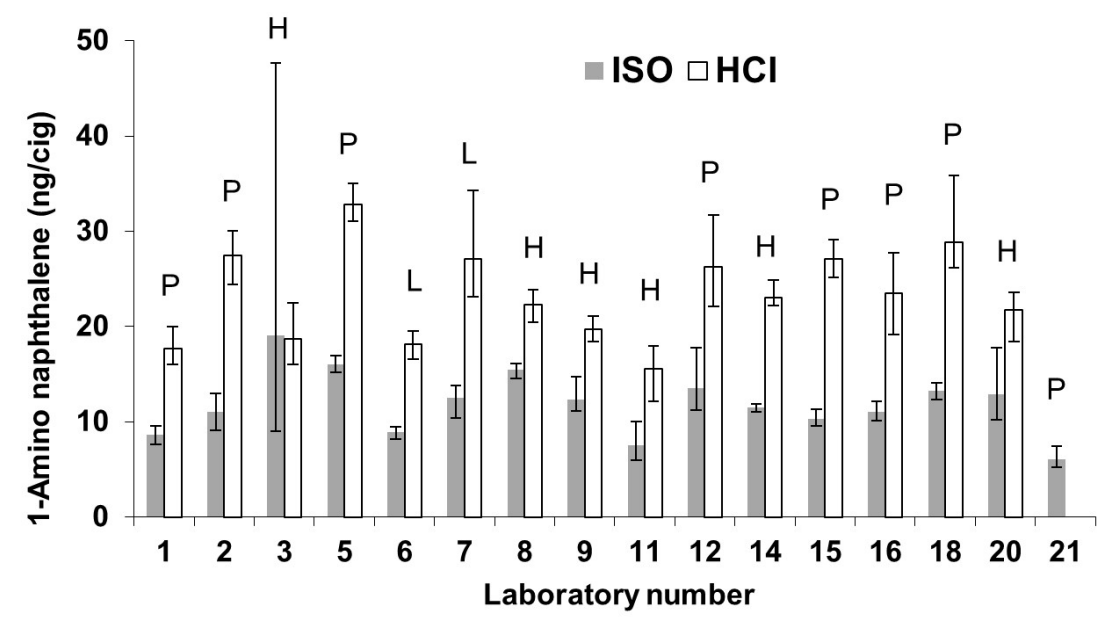

Figure 2. 1-Amino naphthalene yields in Kentucky 3R4F mainstream smoke. $P=P F P A$ reagent; $H=H F B A$ reagent; $L=L C-M S$ method. Maximum and minimum yield values from the 10 replicates are given in the "error" bars.

\section{Hydrogen cyanide}

Hydrogen cyanide yields across the participating laboratories are shown in Figure 1 and the actual values in Tables 9 and 10 under ISO and HCI smoking respectively. The method used by Laboratory 4 gave the lowest yields where they had collected smoke on sodium hydroxide treated CFP with no impingers attached followed by derivatisation to develop colour that could be measured by UV absorbance after CFA analysis. All other laboratories collected on a combination of CFA and impingers then used CFA analysis to give similar results. Laboratories 3 and 9 collected smoke in two impingers then analysed smoke by LCMS/MS which gave similar yields to CFA methodology. Efficient and optimal trapping may be more important than the derivatisation method used although this will need to be confirmed in future joint experiments.

It was observed that the between laboratory variability of hydrogen cyanide was relatively low according to the coefficients of variation given in Tables 9 and 10 and that this should be further reduced during future method standardisation.

\section{Selected aromatic amines}

There were no obvious yield differences observed between the main methodologies involving either PFPA or HFBA derivatisation or LC separation as shown in Figures 2 and 3. Further work is required in order to agree on a method for standardisation. Tables 9 and 10 indicate that there were similar levels of between laboratory variability for ISO and HCI regimes and that the level was relatively higher than for some other of these studied analytes (phenols and hydrogen cyanide). Similar ratios of the four aromatic amines in each laboratory were also observed (see Figure 4) although Laboratories 2 and 11 appear to give inconsistent ratios. 


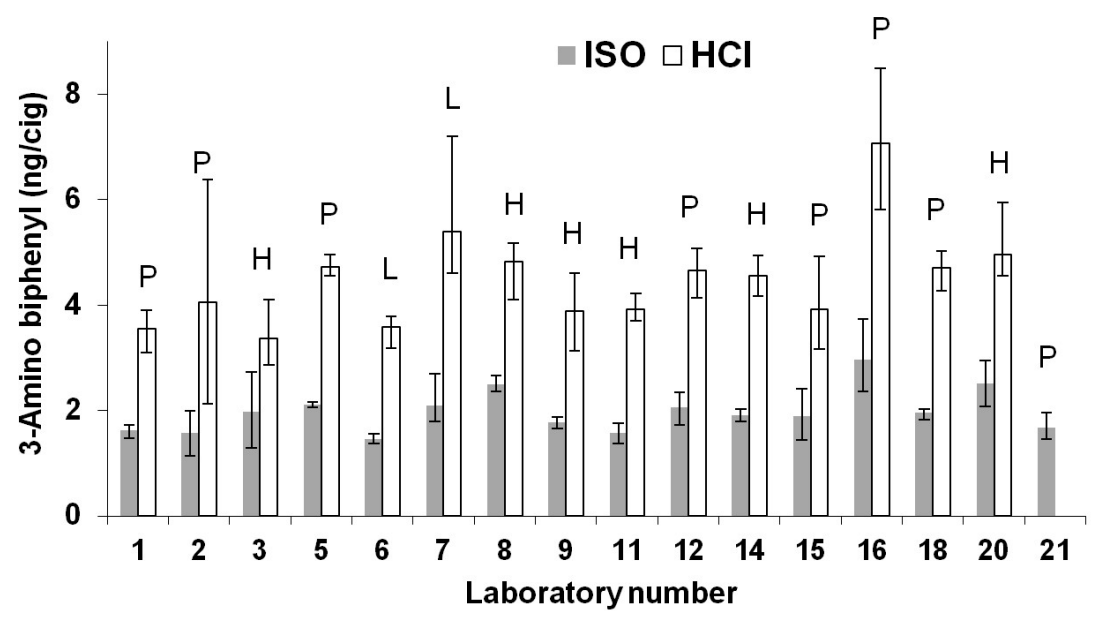

Figure 3. 3-Amino biphenyl yields in Kentucky 3R4F mainstream smoke. $P=P F P A$ reagent; $H=H F B A$ reagent; $L=L C-M S$ method. Maximum and minimum yield values from the 10 replicates are given in the "error" bars.
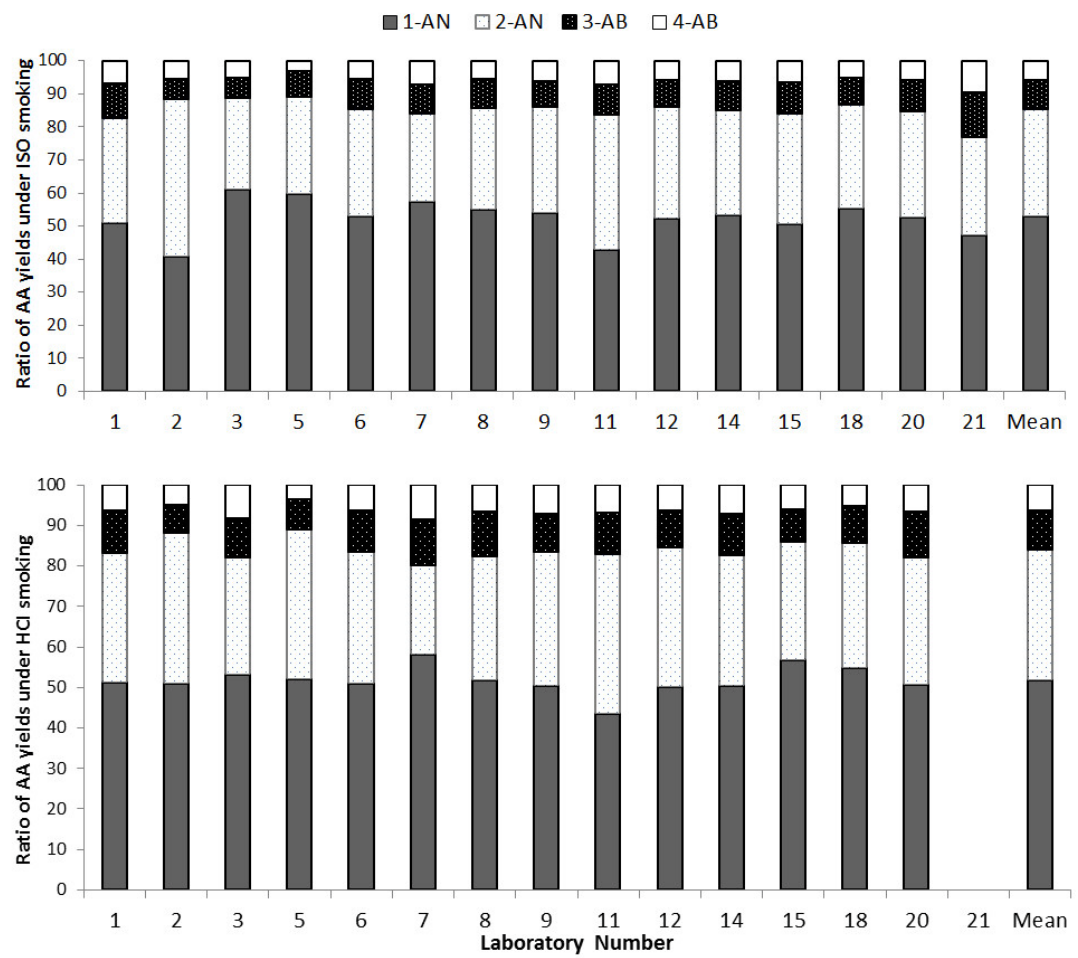

Figure 4. Ratio of aromatic amine yields in Kentucky $3 R 4 \mathrm{~F}$ mainstream smoke under ISO and $\mathrm{HCl}$ smoking regimes. 1-AN = 1-amino naphthalene; 2-AN = 2-amino naphthalene; 3-AB = 3-amino biphenyl; 4-AB = 4-amino biphenyl.

\section{Ammonia}

There was no notable effect of either the use of linear or rotary machine for smoke collection or between the use of different methodologies, that is, ion chromatography separation with conductivity detection or derivatisation then separation and detection of ammonia by LC/MS-MS. Figure 5 shows that the yield variability is in the range of other analytes and so the SPA SG will look to produce a recommended method based on the commonly used ion chromatography method. Tables 9 and 10 indicate that there were similar levels of between laboratory variability for both ISO and HCI regimes.

It has been observed that more efficient IC columns can separate ammonium from interfering ions such as sodium. Artefactual ammonia can be formed from other smoke components (nitriles / amides) by the acid in the collection and extraction steps (27). Laboratories set their autosampler at different temperatures. It has been previously observed that cooling helps to reduce artefacts.

\section{Selected phenolic compounds}

Tables 9 and 10 demonstrate that for these studied analytes, the phenolic compounds had the relatively low levels of variability between laboratories so this should be further reduced during standardisation of the methodology. Similar levels of variability were observed under both the ISO and 


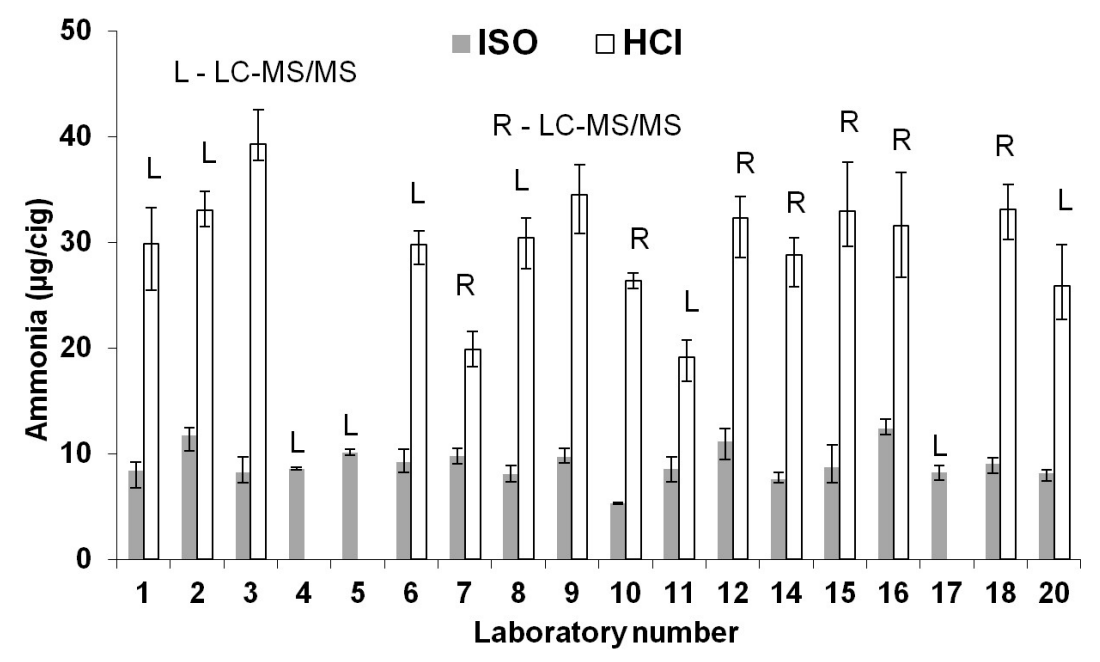

Figure 5. Ammonia yields in Kentucky 3R4F mainstream smoke. $\mathrm{L}=$ linear and $\mathrm{R}=$ rotary smoking machine. Most laboratories used IC / Conductivity. Three labs used LC-MS/MS = liquid chromatography - mass spectrometry / mass spectrometry as indicated. Maximum and minimum yield values from the 10 replicates are given in the "error" bars.

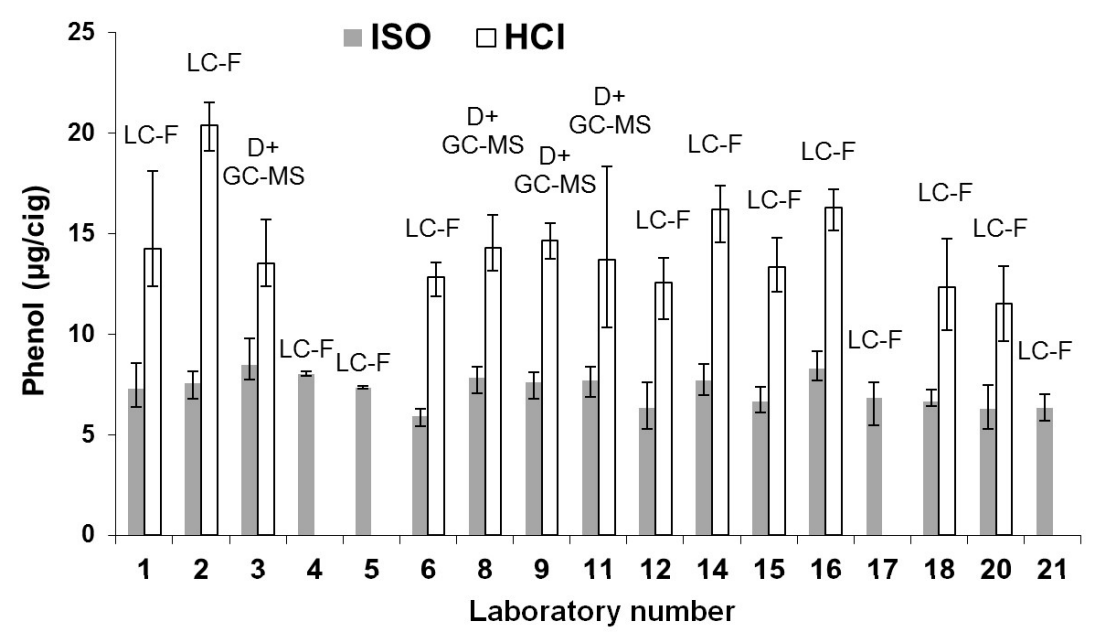

Figure 6. Phenol yields in Kentucky 3R4F mainstream smoke. LC-F = liquid chromatography separation followed by fluorescence detection; D + GC-MS = derivatisation followed by gas chromatography separation and mass spectrometric detection. Maximum and minimum yield values from the 10 replicates are given in the "error" bars.

HCI regime. The LC method with fluorescence detection was used as the basis for joint experiments and collaborative studies working towards a CRM. The use of more efficient reversed phase chromatography columns allows the separation of the meta- and para-cresol isomers. Examples of yield variability for phenol and hydroquinone are shown in Figures 6 and 7. The ratios between the compounds were similar between laboratories in cases when all seven were measured as shown in Figure 8.

\section{Selected semi-volatile compounds}

Tables 9 and 10 provide yield data for pyridine, quinoline and styrene from 3R4F cigarettes. These semi-volatiles collect in substantial amounts within both the CFP and attached impingers and future work will need to address efficient trapping systems. An example for pyridine yields is given in Figure 9. Although Laboratory 2 gave low yields, there were no obvious yield differences found in this study, which can easily be attributed to methodology differences.

\section{Selected metals}

These analytes can be detected in tobacco but they are often below the limits of quantification or detection in smoke due to their low transfer levels and the fact that they are not formed during the smoking process. 3R $4 \mathrm{~F}$ yields are given in Tables 9 and 10. Under ISO smoking conditions, for chromium, there were 4 measured values and 3 below limits of quantitation (BLOQ); for nickel there were 3 measured values and 4 BLOQ and for cobalt there were 4 determinations all below the BLOQ. Likewise, for laboratories carrying out this determination under HCI smoking conditions, for chromium, there were 2 measured values and 4 BLOQ; for nickel there were 3 measured values and 3 BLOQ and for cobalt there were 4 determinations all below the BLOQ.

Optimisation of the digestion step, which removes the interfering organic matrix, needs to be carried out as a major step towards method harmonisation. Results of current CORESTA collaborative studies to develop a CRM on 


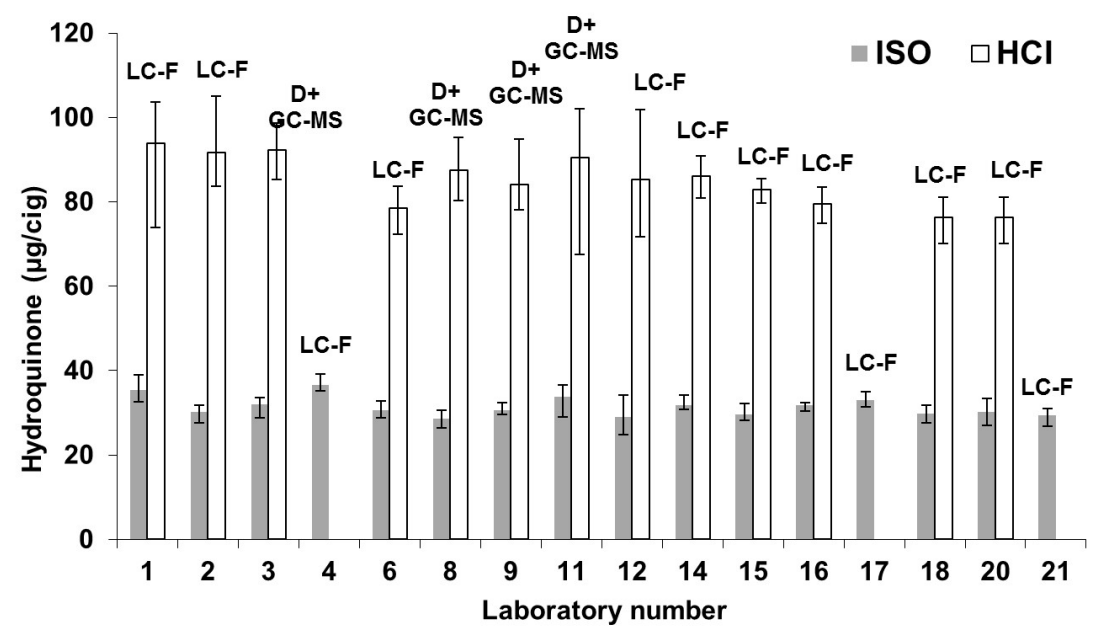

Figure 7. Hydroquinone yields in Kentucky 3R4F mainstream smoke. LC-F = liquid chromatography separation followed by fluorescence detection; D + GC-MS = derivatisation followed by gas chromatography separation and mass spectrometric detection. Maximum and minimum yield values from the 10 replicates are given in the "error" bars.
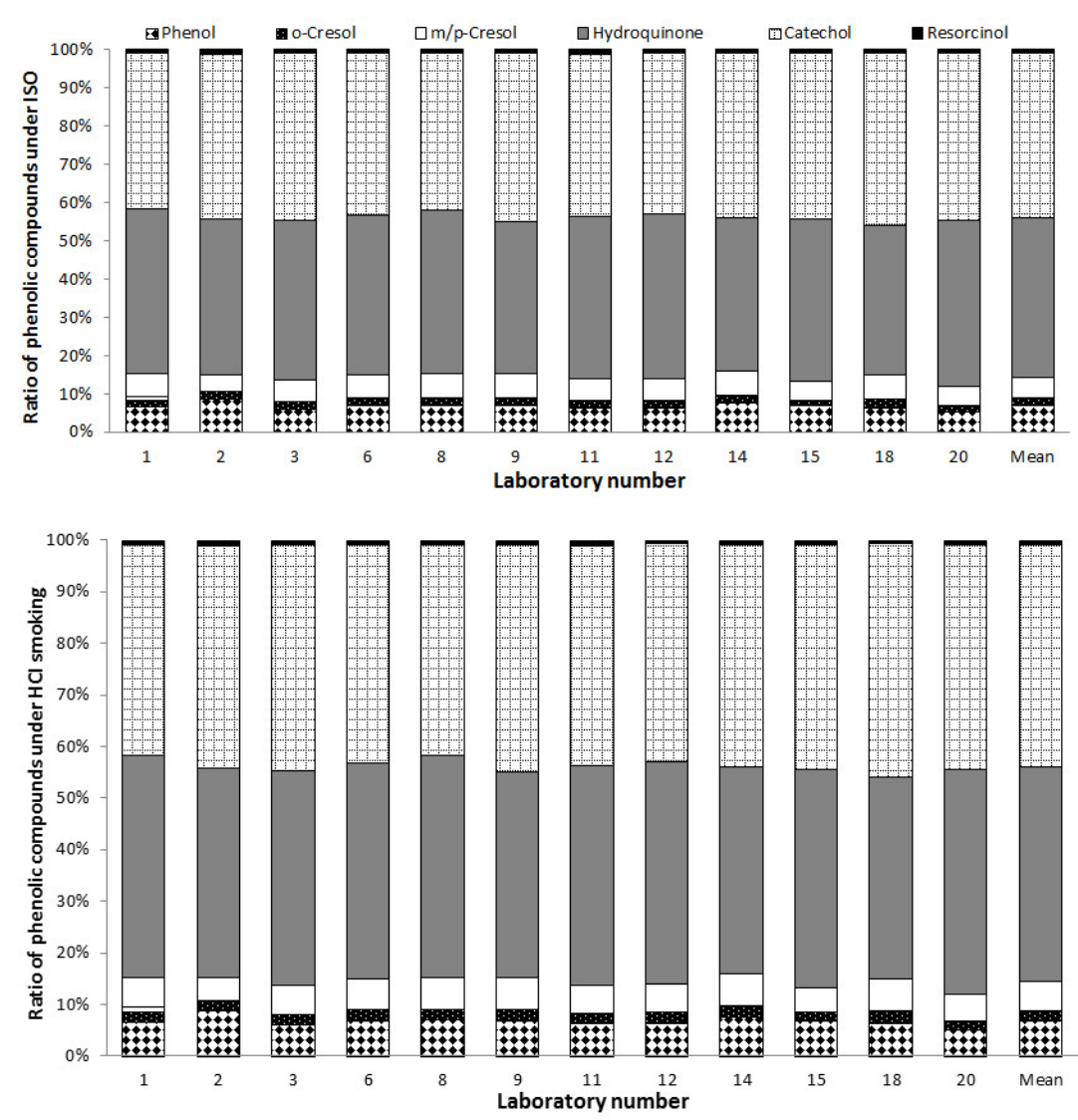

Figure 8. Ratio of phenolic yields in Kentucky 3R4F mainstream smoke under ISO and $\mathrm{HCl}$ smoking regimes.

unburned tobacco will be studied before embarking on the more complicated analyses within the smoke complex.

The most data were obtained on cadmium, lead, arsenic and mercury. Lead yields were more variable than cadmium (see Figures 10 and 11) and this may be due to low level environmental contamination during various handling and analytical stages.
Some recommendations were put forward during SPA SG discussions to reduce between laboratory variability which will need to be considered during CRM development. The challenge will be to define smoke collection and method validation protocols that are appropriate for metals below or near reporting limits and which take account of the impact of contamination. 


\section{CONCLUSIONS}

This study has demonstrated the wide yield ranges obtained by different methodologies and also some of the potential parameters that can cause these differences. As a consequence of this study, the CORESTA SPA SG decided to prioritise the development of CRMs for selected phenols followed by hydrogen cyanide and ammonia.

\section{ACKNOWLEDGEMENTS}

A summary of the 21 participating laboratories in the collaborative study is given below. The Special Analytes Sub Group co-ordinators wish to acknowledge the contributions of each principal investigator and their colleagues without whom the work could not have been completed.

\section{LIST OF PARTICIPANTS}

\begin{tabular}{|c|c|c|}
\hline Participating laboratories & Country & Principal investigator(s) \\
\hline Altria & USA & Karl Wagner \\
\hline Arista Labs & USA & $\begin{array}{l}\text { Alex Martin, } \\
\text { Fraser Williamson }\end{array}$ \\
\hline Arista Labs Europe & UK & Tatiana Humphries \\
\hline British American Tobacco & Brazil & Waldenir Farias \\
\hline British American Tobacco & UK & Jana Ticha \\
\hline CNTC Beijing & China & Jun Zhou \\
\hline CNTC ZTRI & China & Fuwei Xie \\
\hline CNTC Anhui & China & Ran Wang \\
\hline Enthalpy & USA & Gene Gilman \\
\hline Filtrona & UK & Mike Taylor \\
\hline Imperial Tobacco Group & France & Sandrine Destruhaut \\
\hline Imperial Tobacco Group & Germany & $\begin{array}{l}\text { Sandra Otte, } \\
\text { Michael Intorp }\end{array}$ \\
\hline Japan Tobacco & Japan & Takatsuga Hyodo \\
\hline $\begin{array}{l}\text { Japan Tobacco } \\
\text { International }\end{array}$ & Austria & $\begin{array}{l}\text { Jutta Pani, } \\
\text { Alexander Hauleithner }\end{array}$ \\
\hline KT\&G & Korea & John Tae Lee \\
\hline Labstat & Canada & Pete Joza \\
\hline Lorillard & USA & Rob Stevens \\
\hline $\begin{array}{l}\text { Philip Morris International } \\
\text { Operations }\end{array}$ & Switzerland & Guy Jaccard \\
\hline $\begin{array}{l}\text { Philip Morris International } \\
\text { R\&D }\end{array}$ & Switzerland & Michel Rotach \\
\hline Philip Morris Sampoerna & Indonesia & $\begin{array}{l}\text { Wisnu Mahendra, } \\
\text { Rine Raranta }\end{array}$ \\
\hline R J Reynolds & USA & Janell Rowe, Brad Rhoades \\
\hline
\end{tabular}

\section{REFERENCES}

1. World Health Organization (WHO): The Scientific Basis of Tobacco Product Regulation. Second Report of a WHO Study Group; WHO Technical Report Series 951, ISBN $97892 \quad 4 \quad 1209519$ World Health Organization, Geneva, Switzerland, 2008.

2. CORESTA: CORESTA Recommended Method No 58 - Determination of Benzo[a]pyrene in Cigarette Mainstream Smoke by GC-MS; CORESTA, March
2013, available at: http://www.coresta.org (accessed May 2014).

3. CORESTA: CORESTA Recommended Method No 63 - Determination of Tobacco Specific Nitrosamines in Cigarette Mainstream Smoke - GC-TEA Method; CORESTA, June 2005, available at: http://www. coresta.org (accessed May 2014).

4. CORESTA: CORESTA Recommended Method No 75 - Determination of Tobacco Specific Nitrosamines in Cigarette Mainstream Smoke by LC-MS/MS; CORESTA, June 2012, available at: http://www. coresta.org (accessed May 2014).

5. CORESTA: CORESTA Recommended Method No 70 - Determination of Selected Volatile Organic Compounds in the Mainstream Smoke of Cigarettes by GC-MS; CORESTA, March 2013, available at: http://www.coresta.org (accessed May 2014).

6. CORESTA: CORESTA Recommended Method No 74 - Determination of Selected Carbonyls in the Mainstream Cigarette Smoke by HPLC; CORESTA, March 2013, available at: http://www.coresta.org (accessed May 2014).

7. International Organisation for Standardisation (ISO): ISO 3308:2012. Routine Analytical Cigarette-Smoking Machine - Definitions and Standard Conditions; Fifth edition, ISO, Geneva, Switzerland, 2012.

8. Health Canada: Official Method T-115 Determination of "Tar", Nicotine and Carbon Monoxide in Mainstream Tobacco Smoke; Health Canada, Ottawa, Canada, 1999.

9. Intorp, M., S. Purkis, and W. Wagstaff: Determination of Aromatic Amines in Cigarette Mainstream Smoke: The CORESTA 2007 Joint Experiment; Beitr. Tabakforsch. Int. 24 (2010) 78-92.

10. Intorp, M. and S. Purkis: Determination of Selected Volatiles in Cigarette Mainstream Smoke. The CORESTA 2008 Joint Experiment; Beitr. Tabakforsch. Int. 24 (2011) 174-186.

11. Intorp, M., S. Purkis, and W. Wagstaff: Determination of Selected Volatiles in Cigarette Mainstream Smoke. The CORESTA 2009 Collaborative Study and Recommended Method; Beitr. Tabakforsch. Int. 24 (2011) 243-251.

12. Intorp, M., S. Purkis, and W. Wagstaff: Determination of Carbonyl Compounds in Cigarette Mainstream Smoke. The CORESTA 2010 Collaborative Study and Recommended Method; Beitr. Tabakforsch. Int. 25 (2012) 361-374.

13. Intorp, M., S. Purkis, and W. Wagstaff: Determination of Tobacco Specific Nitrosamines in Cigarette Mainstream Smoke: The CORESTA 2011 Collaborative Study; Beitr. Tabakforsch. Int. 25 (2012) 507-519.

14. Purkis, S., M. Intorp, and A. Hauleithner: Updates of CORESTA Recommended Methods After Further Collaborative Studies Carried Out Under Both ISO and Health Canada Intense Smoking Regimes; Beitr. Tabakforsch. Int. 25 (2013) 700-707.

15. International Organisation for Standardisation (ISO): ISO 22634:2008. Cigarettes - Determination of Benzo[a]pyrene in Cigarette Mainstream Smoke - Method Using Gas Chromatography/Mass Spectrometry; ISO, 


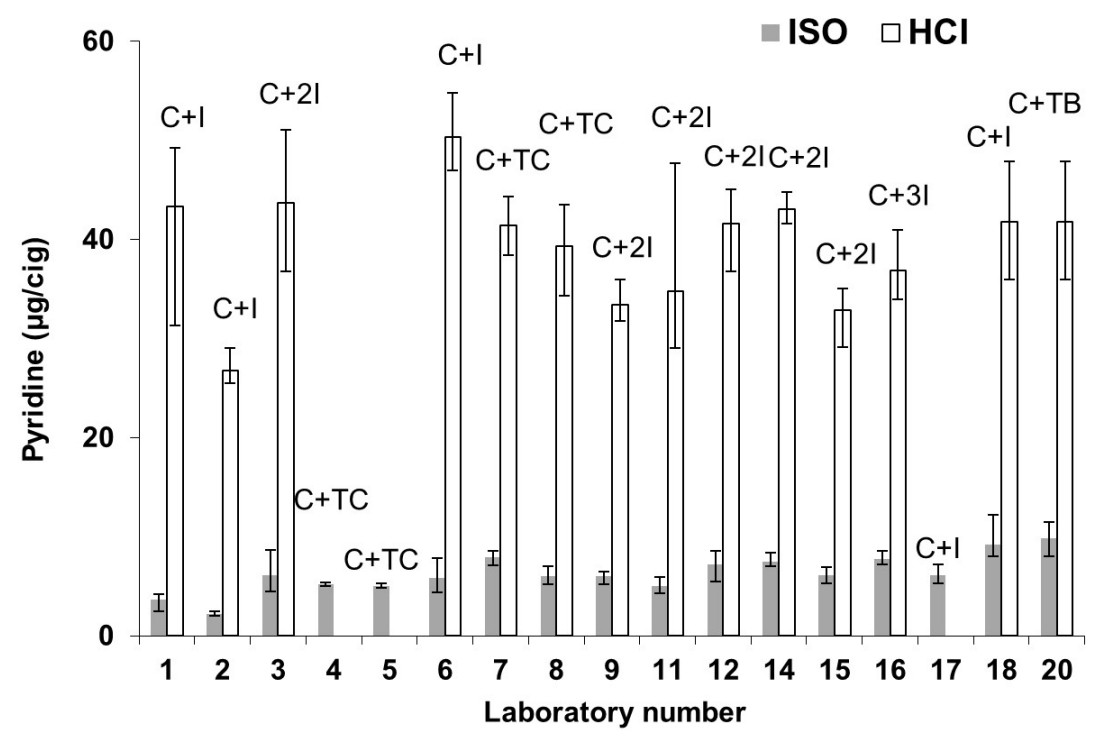

Figure 9. Pyridine yields in Kentucky 3R4F mainstream smoke. $C=$ Cambridge filter pad; $I=$ one impinger; $2 I=$ two impingers; $T C=$ absorbent tube; TB = Tedlar bag. Maximum and minimum yield values from the 10 replicates are given in the "error" bars.

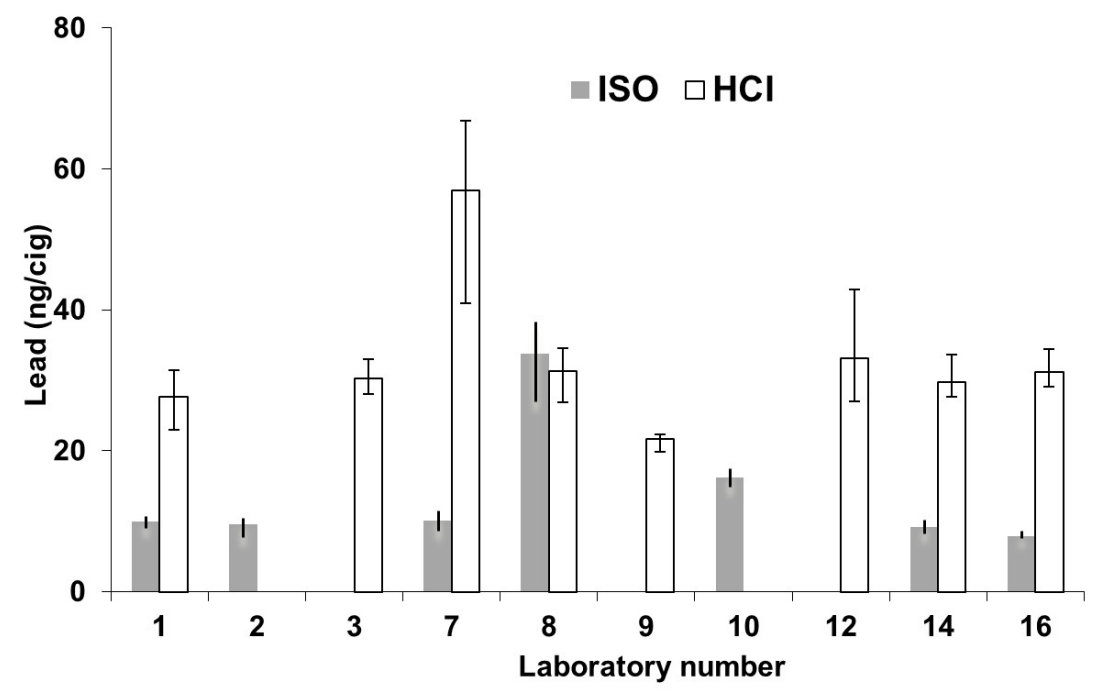

Figure 10. Lead yields in Kentucky 3R4F mainstream smoke. Maximum and minimum yield values from the 10 replicates are given in the "error" bars.

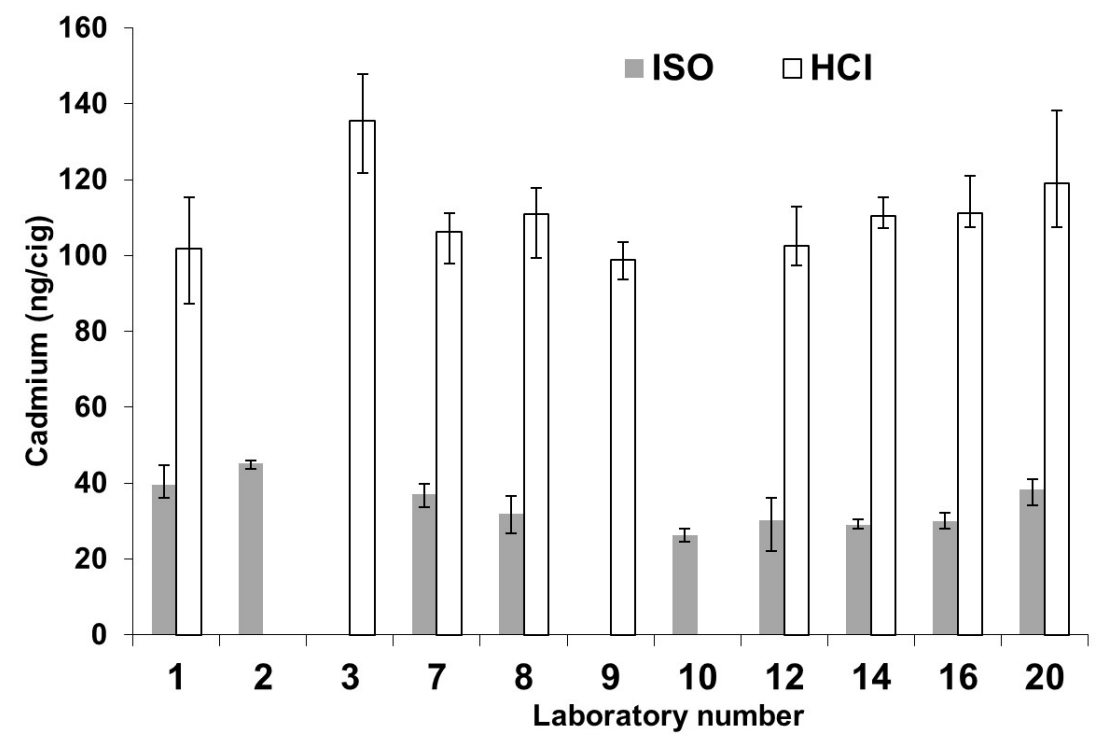

Figure 11. Cadmium yields in Kentucky 3R4F mainstream smoke. Maximum and minimum yield values from the 10 replicates are given in the "error" bars. 
Geneva, Switzerland, 2008.

16. Intorp, M., S. Purkis, M. Whittaker, and W. Wright: Determination of "Hoffmann Analytes" in Cigarette Mainstream Smoke. The CORESTA 2006 Joint Experiment; Beitr. Tabakforsch. Int. 23 (2009) 161-202.

17. Health Canada: Tobacco Reporting Regulations. Regulation June 2000 and the amendments 2005; available at: http://www.hc-sc.gc.ca/hc-ps/tobac-tabac/ legislation/reg/indust/index-eng.php (accessed May 2014).

18. Food and Drug Administration (FDA): Guidance for Industry. Reporting Harmful and Potentially Harmful Constituents in Tobacco Products and Tobacco Smoke Under Section 904(a)(3) of the Federal Food, Drug, and Cosmetic act - Draft Guidance; FDA, Rockville, MD, USA, March 2012, available at: http://www.fda.gov/ downloads/TobaccoProducts/GuidanceComplianceRe gulatoryInformation/UCM297828.pdf (accessed May 2014).

19. Health Canada: Official Method T-107-Determination of Hydrogen Cyanide in Mainstream Tobacco Smoke; Health Canada, Ottawa, Canada, 1999.

20. Ma, L., X.-Y. Li., J. Zhou, Y.-J. Ma, R.-S. Bai, and L.H. Yan: The Influence of Nitrogen Oxides on the Determination of Hydrogen Cyanide in Mainstream Cigarette Smoke by Continuous Flow Analyser; Paper ST19, presented at the CORESTA SSPT Meeting, Graz, Austria, 2011, available at: http://coresta.org (accessed May 2014).

21. Nanni, E.J., M.E. Lovette, R.D. Hicks, K.W. Fowler, and M.F. Borgerding: Separation and Quantitation of Monovalent Anionic and Cationic Species in Mainstream Cigarette Smoke Aerosols by HighPerformance Ion Chromatography; J. Chromatogr. Sci. 28 (1990) 432-436.

22. Mottier, N. and F. Jeanneret: Evaluation of Two Derivatization Reagents for the Determination by LCMS/MS of Ammonia in Cigarette Mainstream Smoke; J. Agric. Food Chem. 59 (2011) 92-97.
23. Risner, C.H. and S.L. Cash: A High-Performance Liquid Chromatographic Determination of Major Phenolic Compounds in Tobacco Smoke; J. Chromatogr. Sci. 28 (1990) 239-244.

24. Nanni, E.J., M.E. Lovette, R.D. Hicks, K.W. Fowler, and M.F. Borgerding: Separation and Quantitation of Phenolic Compounds in Mainstream Cigarette Smoke by Capillary Gas Chromatography with Mass Spectrometry in the Selected-Ion Mode; J. Chromatogr. 505 (1990) 365-374.

25. Houlgate, P.: UK Smoke Constituents Study. Part 11: Determination of Metals Yields in Cigarette Smoke by ICP-MS \& CVAAS; Commissioned by Tobacco Manufacturers Association, London, UK, 2003, available at: http:/www.the-tma.org.uk/benchmark/ benchmarkresources/part11.pdf (accessed May 2014).

26. Tayyarah, R.: Multiple Point in Time Evaluation of Commercial and Reference Cigarette Products for Abbreviated HPHC Yield for Mainstream Smoke and Filler; Paper ST59 presented at the CORESTA SSPT Meeting, September 2013, Seville, Spain, available at: http://coresta.org (accessed May 2014).

27. Kim, H.K., J.T. Lee, K.J. Hwang, and M.S. Rhee: The Change in Ammonium Ion Concentration in the Sample Solution for Smoke Analysis; Paper SS25 presented at the CORESTA Congress, Kyoto, Japan 2004, available at: http://coresta.org (accessed May 2014).

Corresponding author:

Steve Purkis

Imperial Tobacco Limited

Winterstoke Road

Bristol BS3 2LL, UK

E-mail: steve.purkis@uk.imptob.com 